\title{
Noise reduction of a Counter Rotating Open Rotor through a locked blade row
}

DOI:

10.1016/j.ast.2019.105637

\section{Document Version}

Accepted author manuscript

Link to publication record in Manchester Research Explorer

\section{Citation for published version (APA):}

Smith, D. A., Filippone, A., \& Bojdo, N. (2020). Noise reduction of a Counter Rotating Open Rotor through a locked blade row. Aerospace Science and Technology, 98, 105637. [105637]. https://doi.org/10.1016/j.ast.2019.105637

\section{Published in:}

Aerospace Science and Technology

\section{Citing this paper}

Please note that where the full-text provided on Manchester Research Explorer is the Author Accepted Manuscript or Proof version this may differ from the final Published version. If citing, it is advised that you check and use the publisher's definitive version.

\section{General rights}

Copyright and moral rights for the publications made accessible in the Research Explorer are retained by the authors and/or other copyright owners and it is a condition of accessing publications that users recognise and abide by the legal requirements associated with these rights.

\section{Takedown policy}

If you believe that this document breaches copyright please refer to the University of Manchester's Takedown Procedures [http://man.ac.uk/04Y6Bo] or contact uml.scholarlycommunications@manchester.ac.uk providing relevant details, so we can investigate your claim.

\section{OPEN ACCESS}




\title{
Noise Reduction of a Counter Rotating Open Rotor Through a Locked Blade Row
}

\author{
D. A. Smith, A. Filipponef \& N. Bojdo \\ School of MACE \\ The University of Manchester \\ M13 9PL, UK
}

\begin{abstract}
Counter Rotating Open Rotors (CROR) have the potential to significantly reduce aircraft emissions. However, there are a number of concerns surrounding their noise levels. In this contribution, we propose a number of novel configurations to reduce the noise of CROR for general aviation aircraft. In particular, we lock either fore or aft blade row on departure and approach, and keep the CROR trimmed at the same thrust as in design configuration (both rotors running). The CROR is switched back to design configuration at cruise to regain the high propulsive efficiency. To further reduce noise, two additional cases are investigated: 1.) folded aft rotor; 2.) increased diameter fore rotor (with locked-aft blade row). A low-order CROR trim code that has been developed to compute the aerodynamic and aeroacoustic performance of new design configurations is used in the numerical analysis. The results demonstrate significant noise gains for a number of blade count combinations during a constant altitude flyover. The analysis found that using a locked-fore and locked-aft configuration resulted in a maximum noise reduction of around $3 \mathrm{~dB}(\mathrm{~A})$ ( or $3 \mathrm{~dB}(\mathrm{EPNL})$ ) and $3 \mathrm{~dB}(\mathrm{~A}$ ) (or $6 \mathrm{~dB}(\mathrm{EPNL})$ ) respectively. Folding the aft rotor backward could result in a maximum noise gain of around $12 \mathrm{~dB}(\mathrm{~A})$. Increasing the fore rotor diameter by $30 \%$ was shown to reduce noise by a maximum of $8 \mathrm{~dB}(\mathrm{~A})$ against an operative $\mathrm{CROR}$ of the same tip extension.
\end{abstract}

keywords: Counter Rotating Open Rotors, Noise reduction, Low-order, Inoperative rotor

\footnotetext{
${ }^{*}$ Corresponding author. Email: a.filippone@ manchester.ac.uk
} 


\section{Nomenclature}

\section{Roman Symbols}

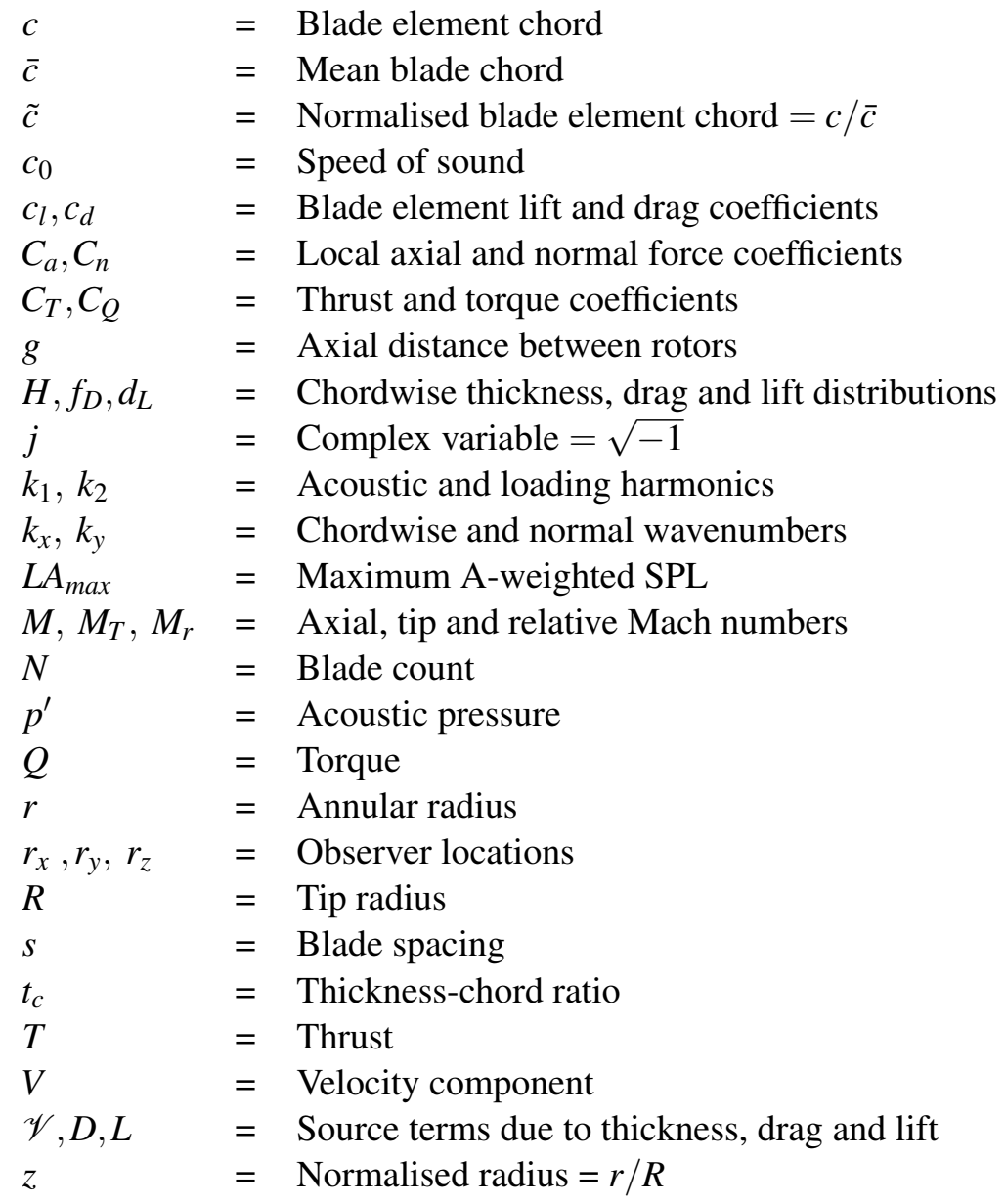

\section{Greek Symbols}

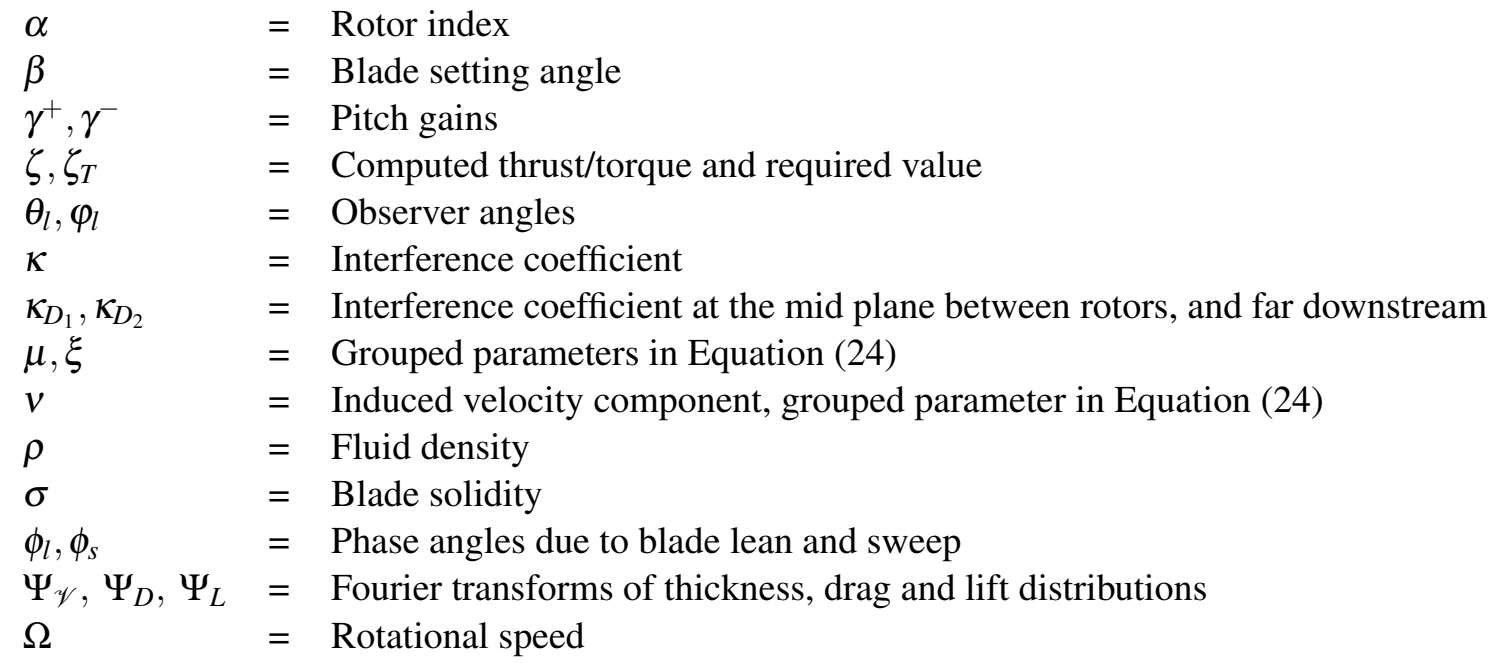




\section{Sub-scripts}

$$
\begin{array}{ll}
{[\cdot]_{x}} & =\text { Axial component } \\
{[\cdot]_{\theta}} & =\text { Tangential component } \\
{[\cdot]_{i}} & =\text { Self-induced component } \\
{[\cdot]_{m i}} & =\text { Mutually-induced component } \\
{[\cdot]_{1,2}} & =\text { Fore/aft rotor } \\
{[\cdot]_{\infty}} & =\text { Free stream }
\end{array}
$$




\section{Introduction}

Counter Rotating Open Rotors (CROR) promise significant fuel savings over modern turbofan engines [1, 2]. The CROR comprises two blade rows rotating in opposite directions around a common axis. Efficiency gains arise due to the high bypass ratio and the removal of residual swirl from the counter rotation of the aft rotor. Prior to integration to modern aircraft, the system will have to comply with all existing and future noise and emissions regulations.

There is a vast patent literature on all matters related to CROR, but one aspect that appears to be missing is the consideration of variable-configuration rotors and their impact on noise. In our study we explore the possibilities of using the CROR as a single rotor in the terminal area in order to reduce noise emissions. These concepts involve locking either fore or aft rotor of the CROR blade pair, with the operative rotor trimmed to deliver the same torque or thrust required by the aircraft. The locked row is then operated to recover the CROR efficiency during cruise. To further reduce noise, we also investigate the case of folding the aft rotor. However, if the noise gains of the folded rotor are minimal over the locked-aft rotor, the latter embodiment is preferable, as it is less mechanically complicated. With the aft rotor still locked, we further explore the advantages, purely from an acoustic point of view, of increasing the fore rotor diameter. For increasing diameters, the tip speed is maintained by reducing the rotation rate of the fore rotor.

The efficiency gains of the CROR were understood from the early days of aviation [3]. The effect of a locked blade row on the aerodynamic performance has also been considered from as far back as the 1940s [4]. There are a limited number of patents concerning a locked or windmilling component, for example Refs. [5, 6], where the importance of feathering the locked component was highlighted to minimise drag and the effects on aircraft handling. However, there is no detail on the impact on noise. In fact, there is no published research on the impact on the noise emissions concerning a locked component of a CROR blade pair. Nonetheless, the literature contains a number of concepts to reduce CROR noise by various means. For example, Zachariadis et al. [7] proposed changes to the rotor rotational speed and setting angles in order to reduce the fore wake contraction and reduce the downstream interaction. Whilst this offered a reduction in interaction noise, a subsequent increase in self noise resulted. Weckmüller \& Guérin [8] demonstrated a noise reduction through the use of serrated trailing edges to increase the mixing of the fore rotor wake. Trailing edge blowing has been investigated by a number of authors [9. 10, 11]. Trailing edge blowing acts to reduce the fore wake deficit and hence reduce the interaction noise. It is also considered to reduce installation effects for pylon mounted configurations [12, 13, 14]. Peters 
\& Spakovszky [15], after quantifying the CROR interaction mechanisms, achieved a reduction in CROR noise with an optimised geometry employing aft rotor clipping and an increased axial spacing. The noise emissions may also be modified by considering unequal rotor rotational speeds [16, 17]. Rotational speeds may be varied in order to increase the destructive addition of the pressure fields from each rotor. Delattre and Falissard [18] investigated the effect of torque ratio to reduce noise. A noise reduction was found when the torque of the aft rotor was greater than the fore. Noise emissions can also be carefully controlled by the fore and aft blade counts [19, 20]. Reductions in noise have also been sought by changing the azimuthal spacing and axially staggering the rotor blades [21, 22]. These concepts are based on changing the phase of the emission of each blade. Both concepts were found to introduce peaks at inter-harmonic frequencies, spreading the peaks over a wider range of frequencies. This would change the perception of the noise for the microphone positions reported; ultimately, it resulted in an increase in sound energy in both cases. These active and passive concepts have demonstrated the potential for reducing CROR noise. In this work, we investigate the use of a variable configuration concept to reduce CROR noise. In particular, a blade row is locked during take-off, climb-out and approach, whilst the design configuration is used in cruise. Therefore, these concepts may offer an attractive solution, with the cruise performance of CROR and reduced noise levels in the terminal area.

Our analysis considers general aviation aircraft (light propeller driven aircraft with a maximum takeoff mass $<8,618 \mathrm{~kg}$ ). However, it may be extended to larger commercial aviation aircraft so long as the operative rotor can provide the required thrust. A number of novel configurations are considered:

- Locked fore rotor

- Locked aft rotor

- Folded aft rotor

- Locked aft rotor with increased fore rotor diameter

These configurations are shown in Figure 1. Each configuration is compared to the baseline CROR, Figure 1(a) in order to demonstrate any gains in terms of noise reduction.

The locked rotor, Figures 1(b) and 1(c), is considered in an attempt to reduce the interaction tones between blade rows. The locking of a rotor blade row will require the operative rotor to provide the required thrust that would typically be supplied by two operative rotors. Hence, the case with an inoperative rotor will be much more highly loaded than the CROR counterpart. As a consequence, the rotor 


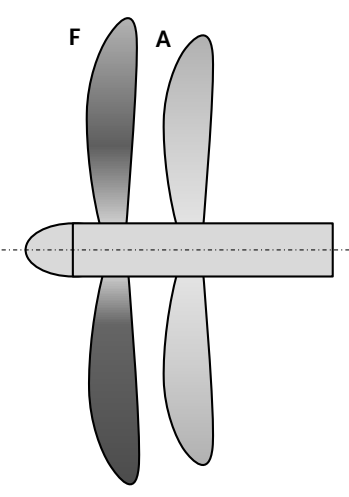

(a) Baseline CROR

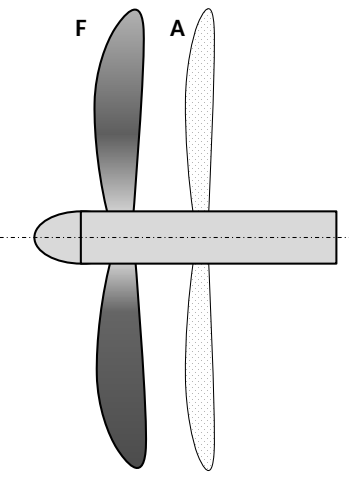

(b) Locked-aft rotor

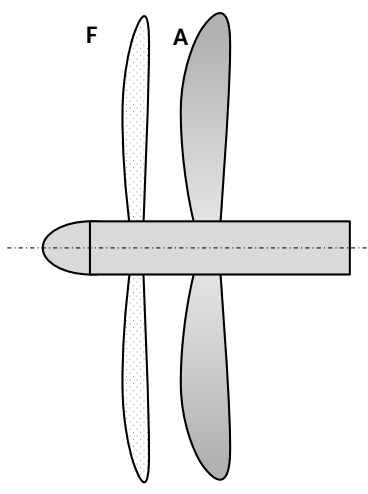

(c) Locked-fore rotor

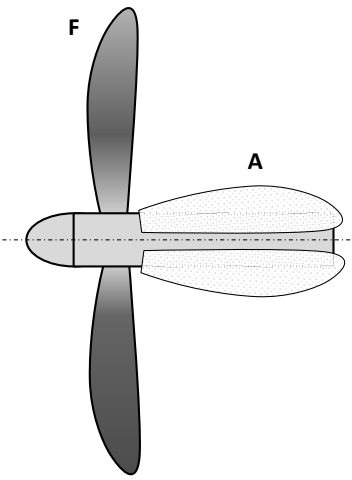

(d) Folded-aft rotor

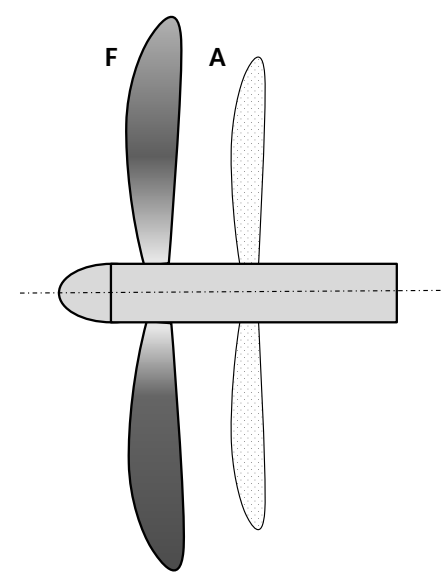

(e) Locked-aft with increased fore diameter

Figure 1: CROR configurations considered in this study. Locked rotors are feathered.

self-noise will increase. There will still exist a number of interaction components between the operative and inoperative rotors. However, these are expected to be much weaker than the baseline CROR. Therefore, a balance must be achieved between increased noise due to loading and the reduction due to interaction tones. As a result, this study focuses on the effects of fore and aft blade count.

In order to reduce interaction tones, the case of the folding aft rotor is also considered, Figure 1(d) There exists a joint at the blade root in order to fold the locked-aft rotor backwards. During cruise, the rotor is unfolded and powered. We only consider the folding mechanism from an acoustic point of view and do not consider the technical design of the device. Again, there will be an increase in loading noise, though in this case the interaction tones should be completely eliminated. The behaviour of the folded rotor will be equivalent to the Single Rotation Propeller (SRP).

The noise gains of the locked component configuration will be limited by the increased loading on the operative rotor. To address this we consider the effect of increasing the fore rotor radius with the locked-aft configuration. We investigate the noise gains by comparing the noise level of the locked-aft and operative CROR configuration with an up to $30 \%$ increase in fore rotor radius. For increasing fore 
radius, the tip speed is maintained by reducing the rotational speed.

In order to prove the potential gains of these concepts, a thorough investigation must be undertaken to demonstrate the ability of the locked rotor to reduce CROR noise whilst providing the required thrust for the aircraft. Gains are demonstrated by comparison with the baseline CROR configuration.

In order to isolate the effects of increasing blade count, the blade solidity is held constant by scaling the blade chord for each case [20]. Therefore, noise reductions are from acoustic criteria only, and hence, we are considering only the aeroacoustic performance of each concept. Future designs must also consider the additional complexities and any design penalties against observed noise gains.

To deal with the large number of parametric cases required for an exhaustive analysis, low-order models have been used. These models offer flexibility and speed of calculation, albeit with a loss of physics. A Blade Element Momentum Theory (BEMT) model extended for CROR [23] is used for the computation of the aerodynamics. Models developed by Hanson [24] \& Parry [25] are used for the computation of CROR noise. They are then adapted to accommodate the case of the inoperative rotor. The locking and unlocking of blade rows is considered instantaneous and no transient effects are considered in the conceptual studies.

In our analysis, we consider the noise emissions in the terminal flight areas. Namely, take-off, climbout and approach conditions. In line with general aviation certification, the maximum A-weighted SPL $\left(L A_{\max }\right)$ noise metric is used throughout the analysis [26]. Certifications procedures require a single microphone measurement at the second segment of take-off. The specific certification altitude is however dependent on the aircraft performance. Therefore, to generalise and to allow for comparisons with climb-out and approach segments, we simulate a constant 1,000 ft (304.8m) flyover to compute the noise metric for each flight segment. Further, a constant flyover allows us to consider only axial flight, somewhat simplifying the analysis.

During the simulated flyover, we consider the CROR in isolation and do not consider any other aircraft noise sources, i.e. we consider CROR noise only. This is justified as we are comparing potential noise gains against a baseline CROR and do not envisage aircraft sources to vary significantly between configurations.

\section{Aerodynamic Models}

The aerodynamic performance of the CROR blade pair was computed using a blade element model extended for the application to CROR [23]. This model allows for quick and accurate computation of 
the induced velocities and blade loads. For each case considered, blade rows were trimmed to give the required thrust at the given flight condition. For cases with an inoperative rotor, the drag due to the inoperative rotor has to be overcome by the operative rotor in addition to the aircraft's required thrust. The inoperative rotor was trimmed to minimise torque.

For the case of the isolated rotor, the radial form of the BEMT equations is applied. These equations are well known and thus their reproduction is unwarranted. The theory is extended to CROR by the inclusion of a number of additional velocity components. These additional components - known as mutually induced velocities — are used to represent the steady interaction between blade rows. The extension of the theory to CROR has been derived earlier [23], and hence only a brief description will be given. The theory is extended by considering an updated schematic of the flowfield, Figure 2.

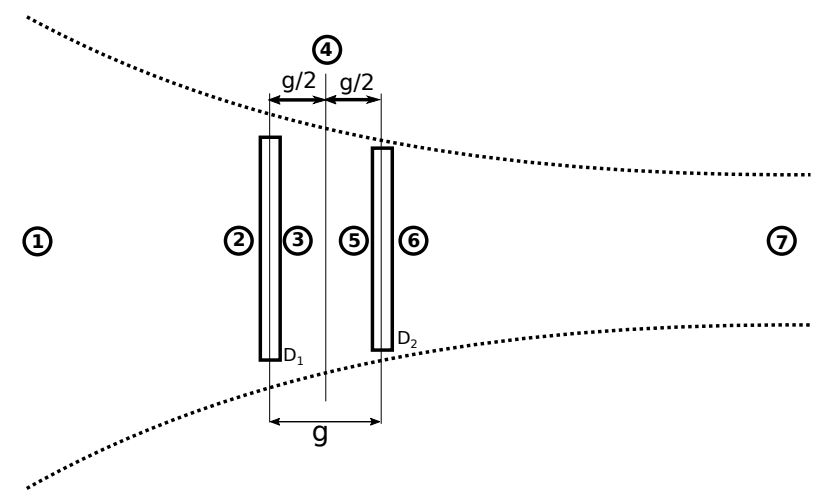

Figure 2: Momentum theory schematic.

From the schematic, the bounding streamtube surrounds both rotors. There is a pressure jump over each rotor which are separated by an axial distance, $g$. The domain comprises a number of discrete zones, where reference is made to a self- and mutually-induced velocity component. The velocity at each of these zones is summarised in Table 1 ,

Table 1: CROR velocity components.

\begin{tabular}{ccc}
\hline Component & Axial & Tangential \\
\hline$V_{1}$ & $V_{\infty}$ & 0 \\
$V_{2}$ & $V_{1}+v_{m i_{x_{1}}}$ & $v_{m i_{\theta_{1}}}$ \\
$V_{3}$ & $V_{2}+v_{i_{x_{1}}}$ & $v_{i_{\theta_{1}}}+v_{m i_{\theta_{1}}}$ \\
$V_{4}$ & $V_{3}+\kappa_{D_{1}} v_{i_{x_{1}}}$ & $V_{3}+\kappa_{D_{1}} v_{i_{\theta_{1}}}$ \\
$V_{5}$ & $V_{3}+v_{m i_{x_{2}}}$ & $V_{3}+v_{m i_{\theta_{2}}}$ \\
$V_{6}$ & $V_{5}+v_{i_{x_{2}}}$ & $V_{5}+v_{i_{\theta_{2}}}$ \\
$V_{7}$ & $V_{5}+\kappa_{D_{2}} v_{i_{x_{2}}}$ & $V_{5}+\kappa_{D_{2}} v_{i_{\theta_{2}}}$ \\
\hline
\end{tabular}

These mutually induced components represent an apparent velocity seen by each rotor due to the opposing rotor. Therefore, the mutually induced components are the product of the self-induced com- 
ponent of the opposite rotor, and an interference coefficient, i.e.:

$$
\begin{aligned}
& v_{m i_{x_{1}}}=\kappa_{x_{21}} v_{i x_{2}} \\
& v_{m i_{x_{2}}}=\kappa_{x_{12}} v_{i x_{1}} \\
& v_{m i_{\theta_{1}}}=\kappa_{\theta_{21}} v_{i \theta_{2}} \\
& v_{m i_{\theta_{2}}}=\kappa_{\theta_{12}} v_{i \theta_{1}}
\end{aligned}
$$

where e.g. $v_{m i_{x_{1}}}$ characterises the effect of the axial velocity of the aft rotor acting on the fore rotor, with analogous definitions for the remaining terms.

The interference coefficients represent the propagation of the self-induced components in the direction towards the opposing rotor. From physical reasoning, results from classical momentum theory and in an attempt to account for the effects of axial spacing between the rotors, the interference coefficients are given by:

$$
\begin{aligned}
\kappa_{x_{12}} & =\frac{g}{2 R_{1}}+1 \\
\kappa_{x_{21}} & =1-\frac{g}{2 R_{1}} \\
\kappa_{\theta_{12}} & =\left(\frac{g}{2 R_{1}}\right)^{1 / 4}+1 \\
\kappa_{\theta_{21}} & =0
\end{aligned}
$$

The additional interference terms $\kappa_{D_{1}}$ and $\kappa_{D_{2}}$ used to calculate the mutually induced terms other than at the rotor plane are simply related to the original terms, as such:

$$
\begin{aligned}
& \kappa_{D_{1}}=\kappa_{x_{21}}\left(g=\frac{g}{2}\right) \\
& \kappa_{D_{2}}=\kappa_{x_{12}}(g=4 R)
\end{aligned}
$$

To account for tip vortex interaction, a simple wake contraction model is applied [27]. For radial elements out-with the contracted wake, the mutually induced terms are set to zero.

From the above expressions of the induced velocity, the momentum theory expressions for thrust and torque can now be developed. To improve computational stability, the equations are developed in their coefficient form:

$$
\mathrm{d} C_{T}=\frac{\mathrm{d} T}{\rho\left(\frac{\Omega}{2 \pi}\right)^{2}(2 R)^{4}}
$$


and

$$
\mathrm{d} C_{Q}=\frac{\mathrm{d} Q}{\rho\left(\frac{\Omega}{2 \pi}\right)^{2}(2 R)^{5}}
$$

The momentum theory thrust coefficient of the fore and aft rotors are given, respectively, by:

$$
\begin{gathered}
\mathrm{d} C_{T_{1}}=\frac{\pi^{3} z_{1}\left(V_{\infty}+\kappa_{x_{21}} v_{i_{x_{2}}}+v_{i_{x_{1}}}\right)\left(\kappa_{x_{21}} v_{i_{x_{2}}}+\kappa_{D_{1}} v_{i_{x_{1}}}\right)}{2\left(\Omega_{1} R_{1}\right)^{2}} \mathrm{~d} z_{1} \\
\mathrm{~d} C_{T_{2}}=\frac{\pi^{3} z_{2}\left(V_{\infty}+\left(1+\kappa_{x_{21}}\right) v_{i_{x_{2}}}+\kappa_{x_{12}} v_{i_{x_{1}}}\right)\left(v_{i_{x_{1}}}\left(\kappa_{x_{12}}-\kappa_{D_{1}}\right)+v_{i_{x_{2}}} \kappa_{D_{2}}\right)}{2\left(\Omega_{2} R_{2}\right)^{2}} \mathrm{~d} z_{2}
\end{gathered}
$$

The torque coefficient for the fore and aft rotors are then given, respectively, by:

$$
\begin{gathered}
\mathrm{d} C_{Q_{1}}=\frac{\pi^{3} z_{1}^{2}\left(\kappa_{D_{\theta_{1}}} v_{i_{\theta_{1}}}-\kappa_{\theta_{21}} v_{i_{\theta_{2}}}\right)\left(V_{\infty}+\kappa_{x_{21}} v_{i_{x_{2}}}+v_{i_{x_{1}}}\right)}{4\left(\Omega_{1} R_{1}\right)^{2}} \mathrm{~d} z_{1} \\
\mathrm{~d} C_{Q_{2}}=\frac{\pi^{3} z_{2}^{2}\left(\kappa_{D_{\theta_{2}}} v_{i_{\theta_{2}}}-\kappa_{D_{\theta_{1}}} v_{i_{\theta_{1}}}\right)\left(V_{\infty}+\left(1+\kappa_{x_{21}}\right) v_{i_{x_{2}}}+\kappa_{x_{12}} v_{i_{x_{1}}}\right)}{4\left(\Omega_{2} R_{2}\right)^{2}} \mathrm{~d} z_{2}
\end{gathered}
$$

with $z_{1,2}$ the normalised blade radius, i.e. $r / R$. For the implementation of the above equations, due to the normalisation with respect to tip speed, the inoperative rotor is given a nominal rotational speed of $10^{-3}$ [rev/min].

With the additional velocity components described above, the extension of the Blade Element Theory (BET) requires the updating of the CROR velocity triangles. The velocity triangles are shown in Figure 3

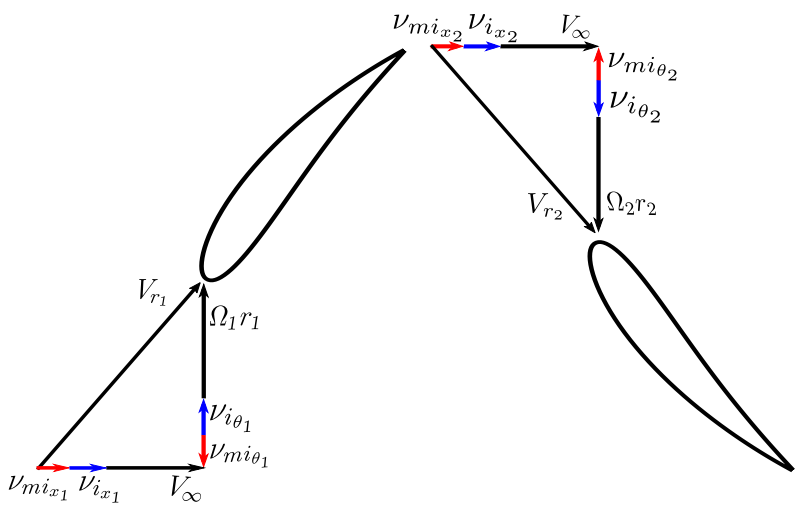

Figure 3: CROR velocity triangles.

The relative velocity at a blade element is then:

$$
V_{r}=\sqrt{\left(V_{\infty}+v_{i_{x}}+v_{m i_{x}}\right)+\left(\Omega r-v_{i_{\theta}}+v_{m i_{\theta}}\right)}
$$


The thrust and torque coefficients are given by:

$$
\begin{aligned}
\mathrm{d} C_{T} & =\frac{\pi^{3} \sigma V_{r}^{2} \tilde{c} C_{a}}{8(\Omega R)^{2}} \\
\mathrm{~d} C_{Q} & =\frac{\pi^{3} \sigma V_{r}^{2} \tilde{c} z C_{n}}{16(\Omega R)^{2}}
\end{aligned}
$$

where $\tilde{c}$ is the chord normalised with respect to the mean chord, $\tilde{c}=c / \bar{c} ; \sigma$ is the blade solidity, $\sigma=$ $N \bar{c} / 2 \pi R$, and $C_{a}$ and $C_{n}$ are the axial and normal force coefficients. These are related to the local twodimensional section lift and drag coefficients:

$$
\left[\begin{array}{c}
C_{a} \\
C_{n}
\end{array}\right]=\left[\begin{array}{cc}
\cos \phi & -\sin \phi \\
\sin \phi & \cos \phi
\end{array}\right]\left[\begin{array}{l}
c_{l} \\
c_{d}
\end{array}\right],
$$

With, $\phi$ the local inflow angle:

$$
\phi=\tan ^{-1}\left(\frac{V_{\infty}+v_{i_{x}}+v_{m i_{x}}}{\Omega r-v_{i_{\theta}}+v_{m i_{\theta}}}\right) .
$$

The local angle of attack, $\alpha=\beta-\phi$, is then used to compute the sectional aerodynamics.

The local aerodynamics, $c_{l}$ and $c_{d}$ are taken from a look-up table for a given aerofoil section with angle of attack and Mach number. Throughout this work, a simplified model for rotorcraft aerofoils was used for the sectional aerodynamics [28]. A K'arm'an-Tsien compressibility correction and a drag divergence model [29] were also utilised to account for the effects at higher relative Mach numbers.

The above equations are applied to each rotor by substitution of the appropriate velocity components to compute their respective blade loading. In order to provide a robust and reliable solution methodology, the blade element and momentum theories are combined. First, an initial guess is made of the induced velocities, $\vec{v}_{i}^{(0)}$. The thrust and torque are then computed using the BET equations. The blade element and momentum equations are then combined:

$$
\left.d T_{1}\right|_{B E T}-\left.d T_{1}\right|_{M T}\left(v_{i_{x 1}}, v_{i_{x_{2}}}, v_{i_{\theta_{1}}}, v_{i_{\theta_{2}}}\right)=0
$$

resulting in a system of non-linear equations. To solve, an inner iteration loop employing a LevenbergMarquardt algorithm [30] is used to solve the system of equations for the induced velocity components. An outer iteration using an under-relaxed successive substitution scheme [31] is then used to compute the thrust and torque using BET. Each iteration loop is exited when the error between successive computations has met a given tolerance. 


\subsection{Rotor Trim}

In order to fully compare the effects of the inoperative rotor with the baseline CROR and SRP, each configuration was trimmed to give the required thrust for the given flight condition. To achieve this, the blade setting angle was successively altered until the thrust or torque produced was within the tolerance of the required value. The change in setting angle was computed according to:

$$
\begin{array}{ll}
\beta=\beta+\gamma^{+}\left|\zeta-\zeta_{T}\right|, & \zeta<\zeta_{T} \\
\beta=\beta-\gamma^{-}\left|\zeta-\zeta_{T}\right|, & \zeta>\zeta_{T}
\end{array}
$$

where $\zeta$ is the computed thrust or torque, and $\zeta_{T}$ the corresponding required value; $\gamma^{+}$and $\gamma^{-}$are the pitch gains, typically of the order of $10^{-6}$.

\subsection{Model Validation}

The aerodynamic model has been compared with experimental data of a CROR. The total thrust coefficient was first computed for a $0.409 \mathrm{~m}$ diameter, $4 \times 4$ CROR of SR2 design [32]. The SR2 is a straight bladed propeller from the NASA Advanced Turboprop Project [33], used as a baseline for investigations into the effect of sweep. The spanwise geometry of the SR2 propeller is presented in Figure 4

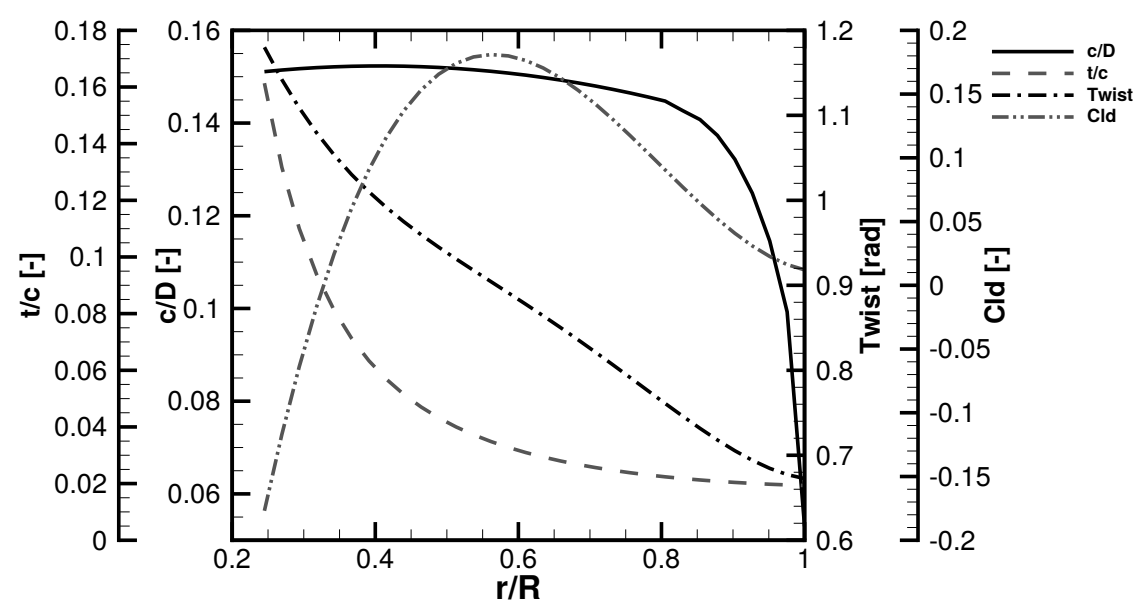

Figure 4: SR2 spanwise geometry.

Figure 5 shows the comparison between the thrust coefficient computed using the BEMT model and the experimental data obtained by Dunham et al. [34]. Results are compared over a range of advance ratios at a fore and aft blade setting angle of $\beta_{.75}=41.34^{\circ}$.

From the comparison with the experimental data, there is a discrepancy at advance ratios $J>1.4$, which could represent a limitation of the aerodynamic model. However, the advance ratios for the work 


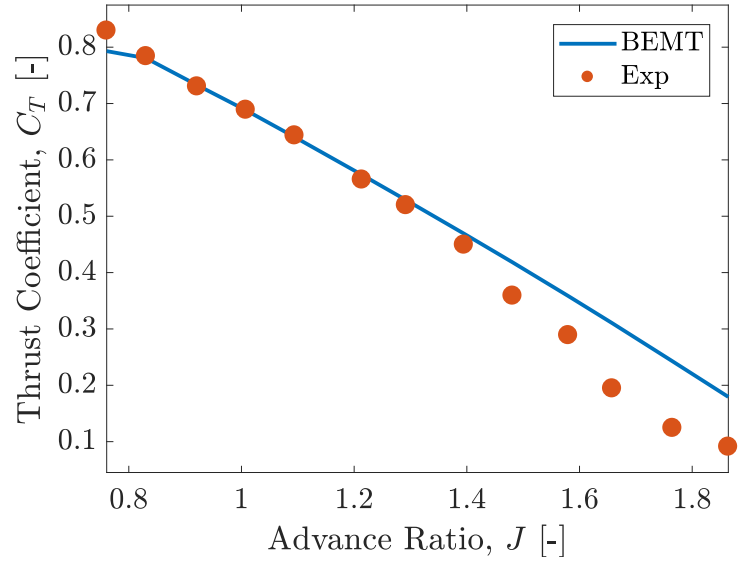

Figure 5: SR2 CROR thrust coefficient comparison.

carried out are $J \simeq 1.0$, which are well within the experimental correlation.

Axial and tangential velocities are then computed at an advance ratio of $J=1.21$ for a number of axial locations. The computed values are shown in Figure 6, where values obtained using BEMT and those obtained by Dunham et al. [34] using laser velocimetry are compared.

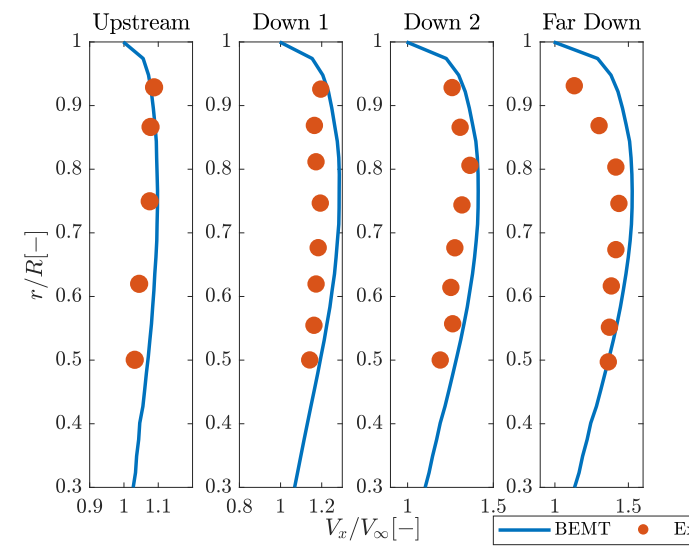

(a) SR2 axial velocity downstream of aft rotor:

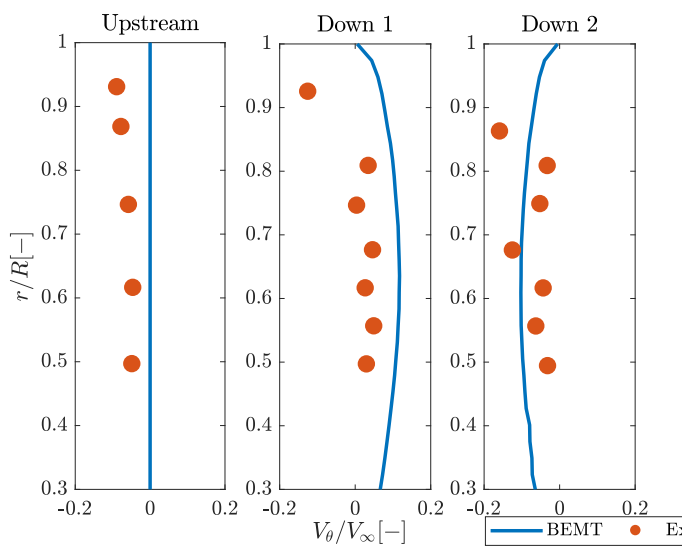

(b) SR2 tangential velocity downstream of aft rotor.

Figure 6: Flow field velocities obtained experimentally and numerically, (a) axial velocity; (b) tangential velocity; advance ratio $J=1.21$.

Figure 6 shows that the aerodynamic model is able to capture well the development of the axial velocity development along the axial direction. Generally, the tangential velocity is well predicted. There are discrepancies due to the lack of a model for the tip vortex. Moreover, there is a discrepancy in the upstream plane as the tangential velocity is assumed to not travel in the upstream direction.

Overall, the aerodynamic model is capable of providing engineering-level performance estimates of the loading and velocity profiles of the CROR blade pair. Given its low computation cost, it is particularly suited for large parametric and preliminary design studies. 


\section{Acoustic Model}

Rotor noise comprises contributions from a number of sources. The acoustic signature can be characterised by steady, unsteady, non-linear and broadband components, as summarised in Figure 7. Steady thickness noise is caused by the periodic volume displacement of the flow surrounding the rotor due to its finite thickness. The steady loading of the rotor, resulting in periodic pressure disturbances, contributes to the steady noise of the rotor. Steady noise is characterised by discrete tones at multiples of blade passing frequency.

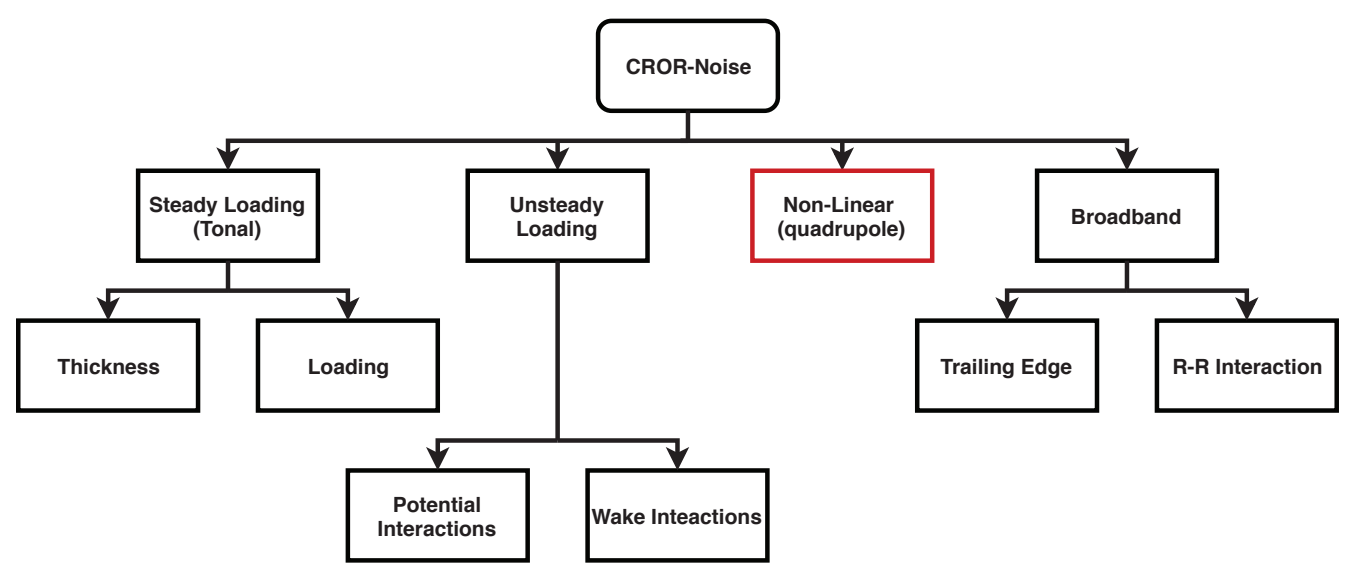

Figure 7: Modelled noise sources. Non linear quadrupole not included in the simulation.

Non-axial flight, ingestion of an upstream pylon wake, installation effects, or in the case of our work, the interaction between blade rows of a CROR blade pair results in unsteady blade loads. These unsteady loads introduce a non-periodic noise source that can contribute significantly to the overall noise signature, particularly for the low-speed operation of CROR. The unsteady noise sources are illustrated in Figure 8. The unsteady noise of a CROR has peaks at interaction harmonics, i.e. the sum and

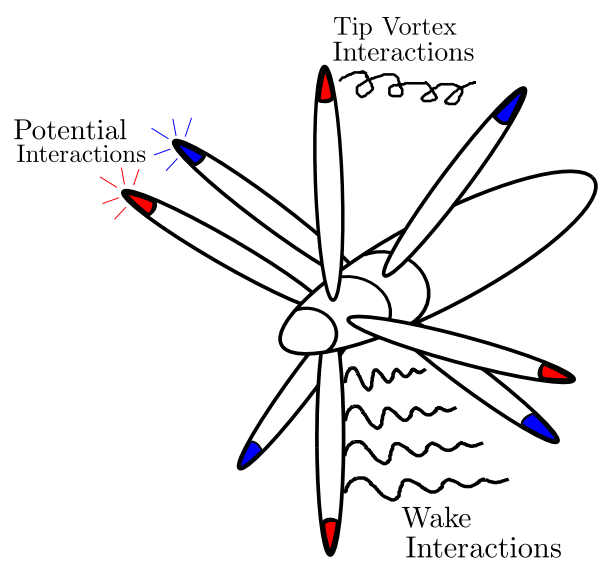

Figure 8: Interactional noise sources.

differences of fore and aft blade passing frequencies. 
When the relative velocity of a rotor blade section starts operating in transonic flow, non-linear noise, a contribution due to blade thickness, can become significant. This can be modelled as a quadrupole source term. However, knowledge of the local transonic flow and perturbations are required, thus making its computation more elaborate.

Finally, small perturbations due to turbulent flow can contribute to rotor noise. The random behaviour of the turbulent flow can contribute to the noise over all frequencies. The major contribution comes from the turbulence at the rotor trailing edge. For the case of the CROR, there can be significant broadband noise due to the rotor-rotor interaction.

Of the above noise sources, all are modelled with the exception of the quadrupole source. This is due to the additional computational effort required to compute the transonic flow perturbations. For general aviation class aircraft, the quadrupole terms should not be significant, due to the low relative blade Mach numbers.

\subsection{Tonal Noise}

The tonal noise can make the most significant contribution to rotor noise, with the peak noise occurring at harmonics of the blade passing frequency. In the case of CROR, the noise also has peaks at interaction harmonics (sum and differences of fore and aft blade passing frequencies), and can still produce high noise levels even at higher harmonics.

\subsubsection{Steady Noise}

The isolated rotor comprises rotor-alone tones from thickness and loading sources. The expression for the acoustic pressure of the rotating rotor due to these components has been reported by many authors [25, 35, 36, 37]. The acoustic pressure for the rotor is read as:

$$
\begin{aligned}
& p^{\prime}(\vec{x}, t)=\frac{-\rho c_{0}{ }^{2} N R}{4 \pi r_{z}\left(1-M_{x} \cos \theta\right)} \sum_{k_{1}=-\infty}^{\infty} \exp \left\{j\left(\frac{k_{1} N \Omega}{1-M_{x} \cos \theta}\left(t-\frac{r_{l}}{c_{0}}\right)+k_{1} N\left(\frac{\pi}{2}-\varphi_{l}\right)\right)\right\} \times \\
& \qquad \int_{z_{h}}^{z_{t}} M_{r}^{2} e^{-j\left(\phi_{l}+\phi_{s}\right)} J_{\left(N k_{1}\right)}\left(\frac{k_{1} N z M_{T} \sin \theta}{1-M_{x} \cos \theta}\right)\left\{\begin{array}{c}
\mathscr{V} \\
D \\
L
\end{array}\right\} \mathrm{d} z
\end{aligned}
$$

The observer locations $r_{z}, r_{l}, \theta_{l}$, and $\varphi_{l}$ are as defined in Figure 9. $k_{1}$ is the acoustic harmonic; $\phi_{l}$ and $\phi_{s}$ 

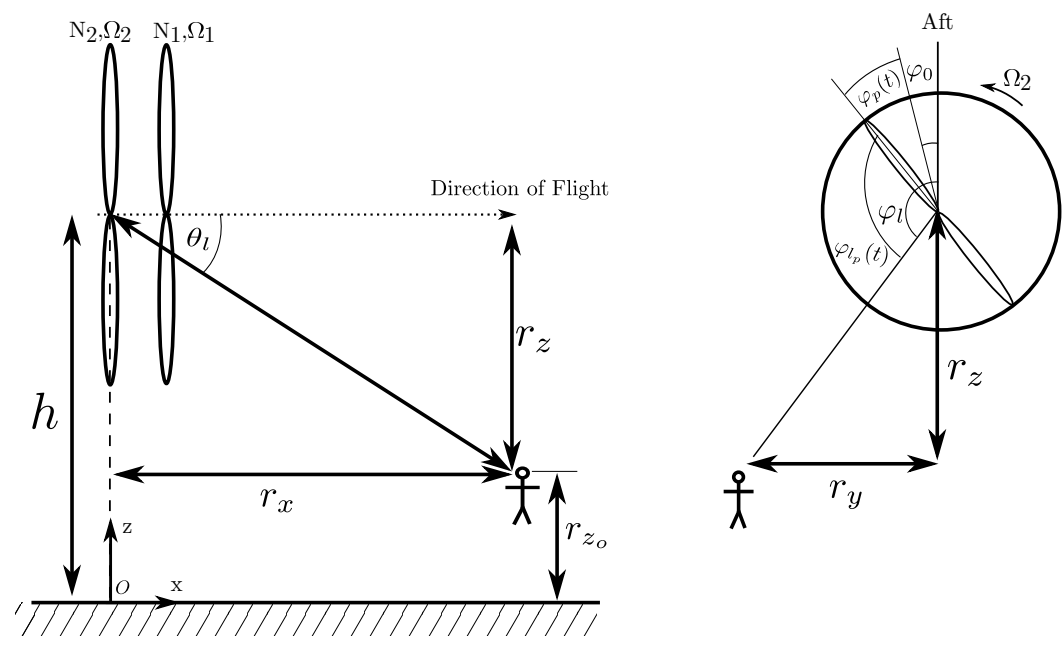

Figure 9: Observer location definitions.

are phase terms due to blade lean and sweep; $J_{v}(Z)$ is a Bessel function of the first kind, of order $v$ and argument $Z . M_{x}, M_{T}$ and $M_{r}$ are the free-stream, tip and relative Mach numbers respectively. The term $\left(1-M_{x} \cos \theta\right)$ is the Doppler frequency shift, with $\theta$ the retarded observer angle; $\mathscr{V}, D$, and $L$, are the source terms due to thickness and drag and lift forces respectively. These are given by:

$$
\left\{\begin{array}{c}
\mathscr{V} \\
D \\
L
\end{array}\right\}=\left[\begin{array}{c}
k_{x}^{2} t_{c} \Psi_{\mathscr{V}} \\
j k_{x} \frac{c_{d}}{2} \Psi_{D} \\
j k_{y} \frac{c_{l}}{2} \Psi_{L}
\end{array}\right]
$$

where $t_{c}, c_{l}$, and $c_{d}$ are the thickness-chord ratio, and lift and drag coefficients respectively. The chordwise wave numbers, $k_{x}$ and $k_{y}$, which represent non-compactness factors are given by:

$$
\begin{aligned}
& k_{x}=\frac{k_{1} N c M_{T}}{R M_{r}\left(1-M_{x} \cos \theta\right)} \\
& k_{y}=\frac{k_{1} N c}{M_{r} r}\left(\frac{M_{r}^{2} \cos \theta-M_{x}}{1-M_{x} \cos \theta}\right)
\end{aligned}
$$

The terms $\Psi_{\mathscr{V}}, \Psi_{L}$, and $\Psi_{D}$ are the Fourier transforms of the thickness, lift and drag chordwise distributions. These are given by [37]:

$$
\left\{\begin{array}{l}
\Psi_{\mathscr{V}} \\
\Psi_{D} \\
\Psi_{L}
\end{array}\right\}=\int_{-\frac{1}{2}}^{\frac{1}{2}}\left\{\begin{array}{c}
H(x) \\
f_{D}(x) \\
f_{L}(x)
\end{array}\right\} \exp \left(j k_{x} x\right) \mathrm{d} x
$$


where $H(x), f_{D}(x)$, and $f_{L}(x)$, describe the thickness and loading distributions along the blade section chord. As the chordwise loading is not available from the BEMT approach, an analytical expression assuming a uniform distribution of lift and drag is used [37]:

$$
\left.\begin{array}{l}
f_{D}(x)=1 \\
f_{L}(x)=1
\end{array}\right\} \quad-\frac{1}{2} \leq x \leq \frac{1}{2}
$$

which gives:

$$
\Psi_{L}=\Psi_{D}= \begin{cases}1 & \left(k_{x}=0\right) \\ \frac{2}{k_{x}} \sin \left(\frac{k_{x}}{2}\right) & \left(k_{x} \neq 0\right)\end{cases}
$$

The assumption of uniform loading should be sufficient for the case here with general aviation CROR [38].

\subsubsection{Unsteady Loading Noise}

Along with the rotor-alone tones, the CROR comprises interaction tones. These consist of acoustic interactions and aerodynamic interactions [24]. Acoustic interactions are due to the constructive and destructive addition of the steady noise components. Aerodynamic interactions are due to the unsteady loading due to up and downstream interactions between blade rows. In the CROR case, unsteady loading arises due to potential interactions, the aft rotor cutting through the wake and tip vortex of the fore rotor. The acoustic pressure on a rotor due to unsteady loading is described by Hanson [24] \& Parry [25] and is given by:

$$
\begin{aligned}
p_{i}^{\prime}(\vec{x}, t)=\frac{\alpha j \rho c_{0}{ }^{2} N_{i} R_{i}}{4 \pi r_{z}\left(1-M_{x} \cos \theta\right)} \sum_{k_{1}=-\infty}^{+\infty} & \sum_{k_{2}=-\infty}^{+\infty} \exp \left\{j\left[\mu\left(t-\frac{r_{l}}{c_{0}}\right)+v\left(\varphi_{l}+\frac{\pi}{2}\right)\right]\right\} \\
& \times \int_{z h_{i}}^{z_{t_{i}}} M_{r_{i}}{ }^{2} e^{j\left(\phi_{s}+\phi_{l}\right)} J_{v}\left[\frac{\xi z_{i} \sin \theta}{1-M_{x} \cos \theta}\right]\left\{k_{y_{i}} \frac{c_{l_{i}}{ }^{\left(k_{j}\right)}}{2} \Psi_{L_{i}}{ }^{\left(k_{j}\right)}\right\} \mathrm{d} z_{i}
\end{aligned}
$$

where

$$
\begin{aligned}
& \mu=k_{1} N_{1} \Omega_{1}+k_{2} N_{2} \Omega_{2} \\
& v=k_{1} N_{1}-k_{2} N_{2} \\
& \xi=k_{1} N_{1} M_{t_{1}}+k_{2} N_{2} M_{t_{2}}
\end{aligned}
$$

The indexes $i$ and $j$, represent the currently considered and the opposite rotors respectively. For the fore rotor, i.e. $i=1, j=2, \alpha=-1$. For the aft rotor, i.e. $i=2, j=1, \alpha=+1$. Again, the observer location definitions are given in Figure 9, note the reference to the aft rotor. Note there are now two 
harmonics. For the fore rotor, $k_{1}$ would be the acoustic harmonic, whilst $k_{2}$ would be the loading harmonic; the opposite is true for the aft rotor. The non-dimensional wave numbers (again characterising non-compactness) are, in this case, given by:

$$
\begin{gathered}
k_{x_{i}}=\frac{c_{i}}{R_{i} M_{r_{i}}}\left[\frac{\xi}{1-M_{x} \cos \theta}-k_{j} N_{j}\left(M_{t_{1}}+M_{t_{2}}\right)\right] \\
k_{y_{i}}=\frac{-\alpha c_{i}}{R_{i} M_{r_{i}}}\left[\frac{\xi M_{t_{i}} z_{i} \cos \theta}{1-M_{x} \cos \theta}+\alpha \frac{v M_{x}}{z_{i}}\right]
\end{gathered}
$$

The theory developed by Parry [25, 39, 40] was used to compute the unsteady loading on each rotor. In particular, the model for potential interactions and the Gaussian model for wake interactions. Tip vortex interaction noise is understood to be a major source of interaction noise for CROR. However, it is not accounted for in this analysis. This is justified as the tip vortex source can be reduced by clipping of the aft rotor [41]. Uniform chordwise loading is again assumed in the computation of the unsteady loading noise.

To compute the unsteady loading on the inoperative rotor, the above expressions for unsteady loading noise are simplified by setting the rotational speed of the rotor of interest to zero, $\Omega_{i}=0$.

\subsection{Broadband Noise}

Broadband noise is typically lower than the tonal component. However, whilst the tonal component appears at discrete frequencies, the broadband component exists over the whole frequency range. The broadband noise results from the scattering of the turbulent boundary layer at the rotor trailing edges. Furthermore, there can be significant broadband noise due to the mutual rotor interaction. The derivation of the expressions for these broadband expressions can be quite lengthy, and their reproduction is unwarranted here. The reader is directed towards the model described by Magliozzi [37] for computation of the trailing edge broadband noise. For the CROR case, the broadband interaction noise model described by Blandeau [42, 43] and Kingan [44] is used.

\subsection{Acoustic Model Validation}

There is limited experimental data in the open literature with which to validate the acoustic models for CROR. Nonetheless, the data that is available has been used where possible. The quality of the data is not known, however, it is used here as the only available source with which to compare the numerical models. Noise measurements taken by Block [45, 46, 47] at the NASA Langley $4 \times 7$ metre wind tunnel 
on scale model SR2 SRP and CROR configurations form the basis of the noise validation.

The case of an isolated 4 bladed SRP is considered at two operating points. The first considers the rotor at a setting angle of $20.6^{\circ}$ rotating at $120 \mathrm{rev} / \mathrm{s}$. In the second case, the rotor is at a setting angle of $12.7^{\circ}$, rotating at $168 \mathrm{rev} / \mathrm{s}$. In both cases, the free-stream velocity is $\sim 30 \mathrm{~m} / \mathrm{s}$. Figures $10(\mathrm{a})$ and 10(b) compare the reported [46] and computed Overall Sound Pressure Level (OASPL) for the two SRP operating cases.

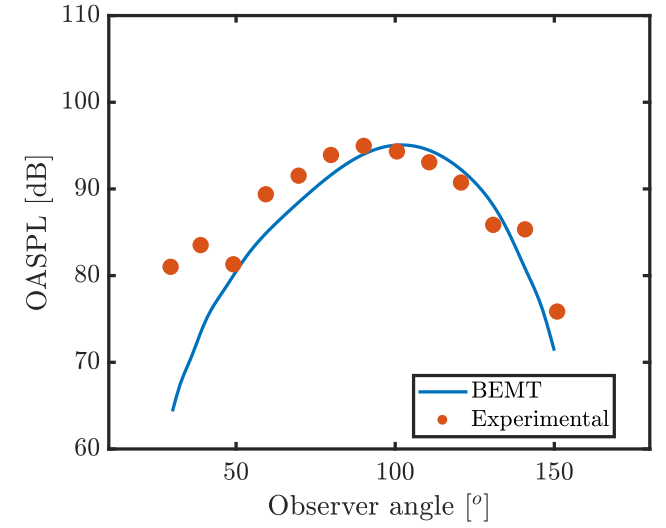

(a) Setting $=20.6^{\circ}, n=120 \mathrm{rev} / \mathrm{s}$.

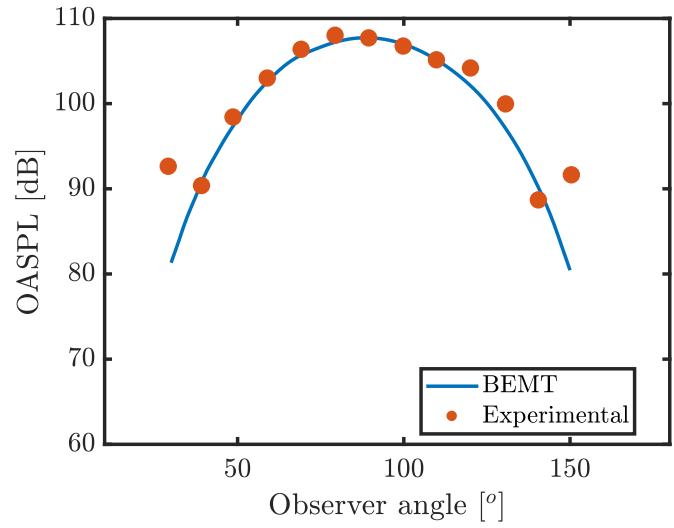

(b) Setting $=12.7^{\circ}, n=168 \mathrm{rev} / \mathrm{s}$.

Figure 10: SRP noise prediction against experiment.

Figure 10 demonstrates that the acoustic model predicts well the OASPL of the SRP in both cases. There are some discrepancies towards the extremes of the observer angles. However, this was also found in numerical comparisons by Block [46], and is described as possibly being caused by nacelle diffraction as the polar angle approaches the rotor axis.

Block [45, 47] also presented noise levels for a $0.409 \mathrm{~m} 4 \times 4$ CROR. Block [45] presented noise measurements of a CROR over a range of azimuth angles at a fixed axial location from the plane of rotation. In this case, both rotors rotated at $\sim 128 \mathrm{rev} / \mathrm{s}$. Figure 11 compares the OASPL levels and the SPL of the first harmonic of BPF between those reported and those computed using the above model.

The predicted azimuthal directivity trends compare well to those reported by Block. Both model and reported data show asymmetric patterns about the $y=0$ position, caused by the axial spacing between blade rows. However, the asymmetrical patterns differ between model and reported data. This results in a discrepancy between the two at a number of points. This discrepancy may indicate deficiency in the way the model accounts for the azimuthal directivity $\left(\varphi_{l}+\frac{\pi}{2}\right.$ term in Equation (23)). Further, the difference in peak values of the reported data may indicate discrepancies in the data. However, as the quality of the data is not known it is difficult to comment.

Block [47] also presented data over a range of polar angles. In this case, both rotors rotated at 


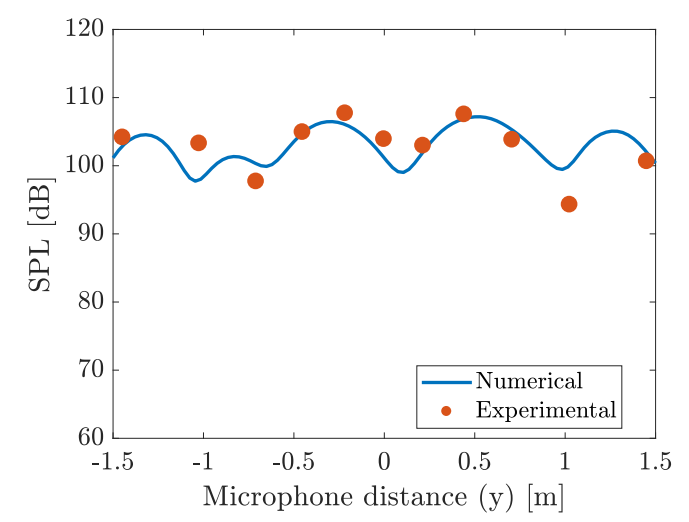

(a) OASPL

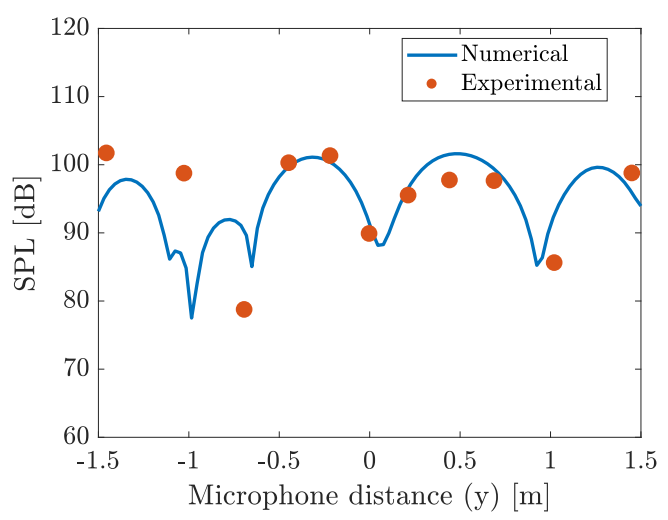

(b) BPF1

Figure 11: CROR azimuthal directivity noise prediction.

$\sim 192 \mathrm{rev} / \mathrm{s}$. The BEMT method was used to trim the rotors to the reported power loading and the noise computed for a range of polar and azimuthal observer angles. Similarly to Block, this was then averaged over the azimuth to give the polar directivity trends. Figure 12 compares the experimental and numerical results for the OASPL and first three harmonics of BPF.

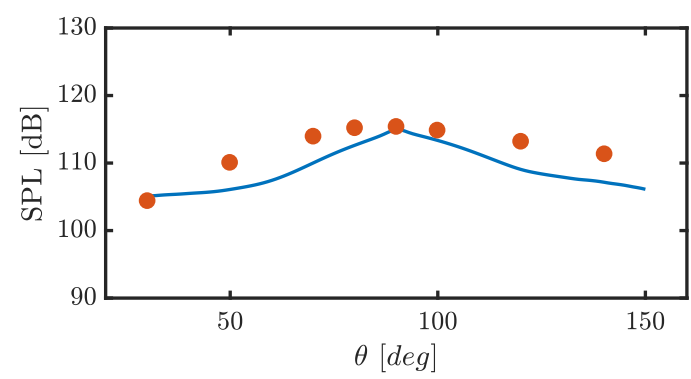

(a) $O A S P L$

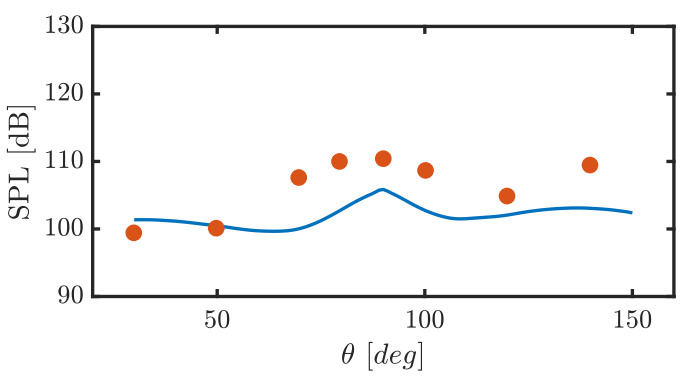

(c) $B P F 2$

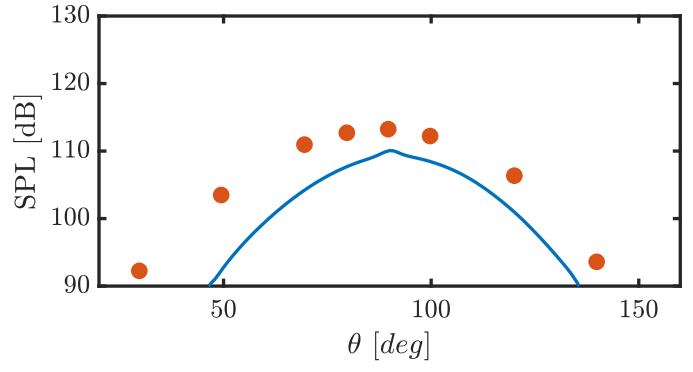

(b) BPF1

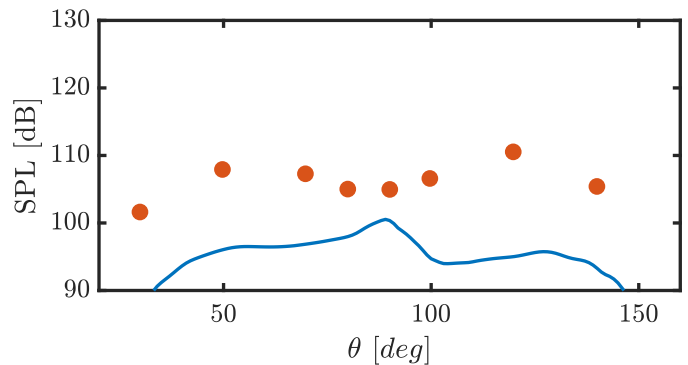

(d) $B P F 3$

Figure 12: CROR noise prediction against experiment.

Figure 12 shows that the OASPL has been predicted relatively well. The first harmonic of BPF is predicted less well. It differs from the reported value at the $90^{\circ}$ polar angle by around $3 \mathrm{~dB}$, with the difference increasing moving away from the overhead position. The second harmonic is predicted well. In particular, the values close to the plane of rotation where the interaction noise will dominate is well predicted. The third harmonic of BPF is not so well predicted, with the peak at the overhead position 
under predicted by around $4 \mathrm{~dB}$. Despite the similar trend near the plane of rotation, the SPL levels are also under predicted. Given the high rotational speed of this case, it was found that the thickness noise dominated the sound levels. Increasing the tip speed by $5 \%$ compared to the reported value showed a marked improvement in the comparisons. However, the accuracy of the test and equipment data are not known and thus it is not possible to verify if this is the cause of the disagreement. The greater discrepancy of the third harmonic may indicate shortcomings of the interaction model used.

In both of the above validation cases, both fore and aft rotors had equal blade counts and tip speeds. Therefore, no interaction tones could be extracted. In order to demonstrate the interaction tone prediction we consider the case with fore and aft rotors rotating at non-equal speeds. Figure 13 presents the noise prediction of rotor alone and the first interaction tone $\left(B P F_{1}+B P F_{2}\right)$.

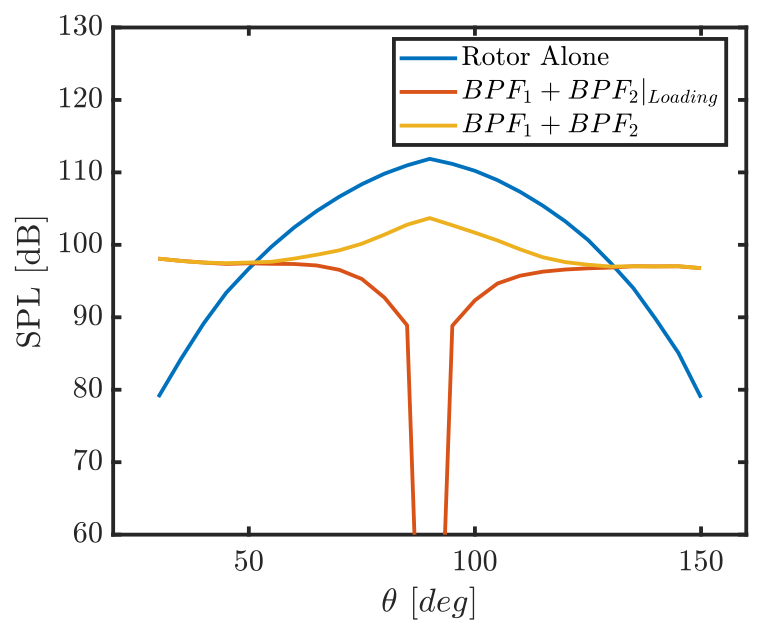

Figure 13: $C R O R$ rotor alone and first interaction $\left(B P F_{1}+B P F_{2}\right)$ tones.

Figure 13 shows the rotor alone tones dominate the spectrum in the region near the rotor plane. Near the plane of rotation, on the other hand, the interaction tone dominates. In Figure 13 the interaction tone has been split into the total and loading components. Due to the high rotation rate, the first interaction tone is dominated by the thickness component near the rotor plane. In this region, the loading component travels as a plane wave along the rotation axis and is shown by the significant drop in noise. Towards the plane of rotation, the interaction noise is dominated by the loading component caused by the unsteady aerodynamic interactions between the rotors.

The models described above been compared to a number of available experimental data sets. Comparisons have shown the model has been able to capture the steady noise of an isolated SRP. The model has been able to capture both azimuthal and polar directivity trends for a CROR model to a suitable level for the analysis carried out here. Whilst the data used for the validation may not be the most representative of the current state-of-the-art CROR and with the accuracy of the data unknown, it remains the only 
available data set that allows for the comparison of CROR noise.

\section{Results and Discussion}

\subsection{Inoperative Fore Rotor}

The fore rotor is locked and trimmed to minimise torque. The operative aft rotor is trimmed to deliver the aircraft thrust requirements in addition to the drag produced by the inoperative fore rotor. The major noise sources will be the rotor self noise and the noise produced from operating in the wake of the locked-fore rotor - analogous to stator-rotor interactions found in compressor noise.

Table 2 shows the rotor design parameters used throughout the analysis. The SR2 propeller geometry (Figure 4) was used for all cases. Clipping of the aft rotor is typically employed in CROR to minimise tip vortex interaction noise [15, 41]. However, the purpose of this work is to investigate a number of novel noise reducing concepts and, therefore, clipping is not employed in this study. Nonetheless, clipping could be used alongside the present concepts to further reduce noise

Table 2: Rotor design parameters.

\begin{tabular}{ccccccc}
\hline Parameter & $s / c$ & $\sigma$ & $R_{1}=R_{2}[\mathrm{~m}]$ & $r_{h} / R$ & $g / R$ & $M_{T}$ \\
\hline Value & 1.25 & 0.18 & 1.5 & 0.24 & 0.4 & 0.46 \\
\hline
\end{tabular}

In order to isolate the effects of increasing blade count and to ensure a physical geometry when blade counts were increased, the blade chord was scaled to ensure constant solidity, this was done by maintaining the rotor spacing-chord ratio. Therefore, for each case, the force per unit blade area is constant for each blade count combination considered. Hence, differences in noise levels arise from acoustic criteria only [20].

The analysis was carried out at take-off, climb-out and approach conditions. These are the most critical phases of flight for the aircraft noise evaluation. Table 3 shows the rotor operating characteristics at each flight phase considered.

Table 3: Operating point parameters.

\begin{tabular}{cccc}
\hline Operating point & Take-off & Climb & Approach \\
\hline Altitude [feet] & 1,000 & 1,000 & 1,000 \\
$M_{x}$ & 0.2 & 0.3 & 0.3 \\
$C_{T}$ & 0.30 & 0.19 & 0.092 \\
\hline
\end{tabular}

These values were obtained using the FLIGHT aircraft performance code [48, 49] for the Beechcraft 350i aircraft to represent a general aviation class aircraft. 
The aircraft was simulated to make a fly-over at a constant 1,000 feet $(304.8 \mathrm{~m})$ altitude, with the observer located at the origin position (see Figure 9) for each flight segment considered. The SPL was then computed at 0.5 second intervals along the fly-over. The noise is then characterised using the maximum A-weighted SPL $\left(L A_{\max }\right)$ from the fly-over. The aerodynamic performance and the resulting noise was computed for a range of blade count configurations from 4 to 6 on the front rotor and 3 to 7 on the aft rotor. These blade counts and combinations were chosen as representative of blade counts of modern propeller driven general aviation aircraft.

Figure 14 shows a comparison of the computed $L A_{\max }$ for the baseline CROR and the case with a locked-fore rotor.

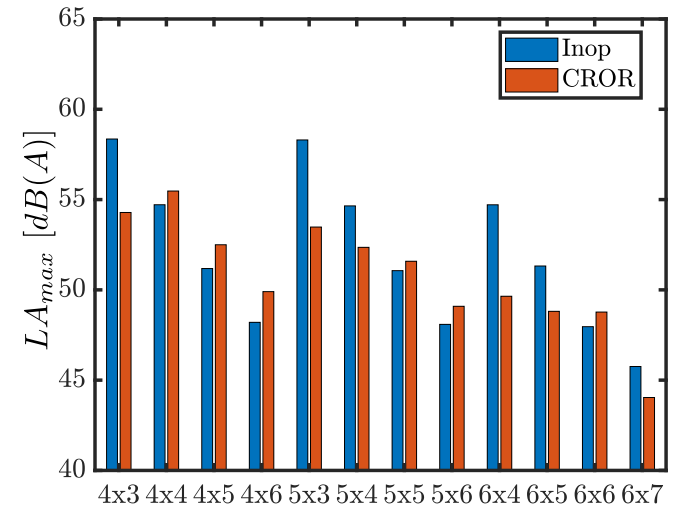

(a) Take-off

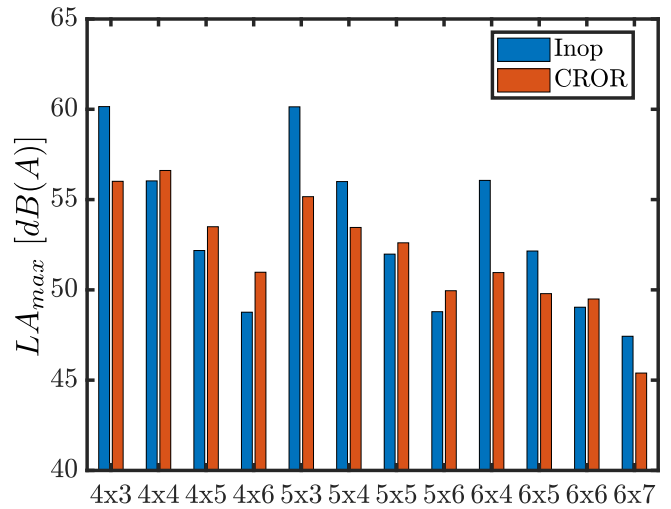

(b) Climb-out

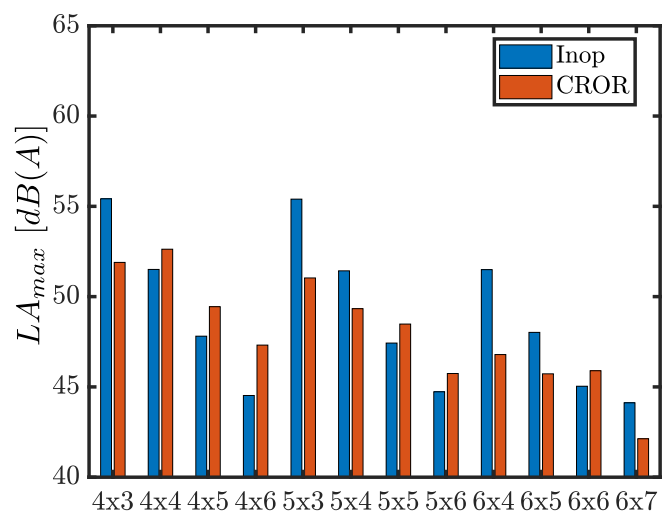

(c) Approach

Figure 14: Comparison of noise between baseline CROR and a CROR with locked-fore blade row.

Figure 14 shows that despite differences in $L A_{\max }$, the trends are consistent through the three operating points. This is due to the dominance of the loading components at these flight phases. During approach conditions in particular, it is understood that other airframe sources may dominate the peak levels measured. However, we are only interested in comparing the noise levels between the baseline and inoperative configurations. 
As the $L A_{\max }$ metric has been used, the differences found between the two configurations are likely due to differences in self noise as this has been found to dominate the noise, in particular near the plane of rotation. Returning to Equation (23), the equation for the noise due to unsteady loading shows, in particular the integral term, that the noise is proportional to some amplitude (part containing the loading, or thickness term, although it is known that the loading will dominate at these low speed operating points) and a radiation efficiency (part containing the Bessel function). For the steady loading case (i.e. $k_{1}=0$ for the aft rotor, $k_{2}=0$ for the fore rotor), both configurations will radiate with the same efficiency. Furthermore, as the loading per unit blade area is held constant (through constant solidity), changes in noise for a given configuration will be due to the acoustic effects of the blade count. Therefore, differences in noise level between the two configurations will arise due to differences in loading between configurations (i.e. the total thrust being produced by a single rotor or by two rotors) and the blade count combination of the operative configuration. This is evidenced in Figure 14, for example considering the fore-locked configuration. Noise levels are comparable for equal aft blade counts. Considering the operative configuration, noise levels are comparable between opposite blade counts, e.g the $5 \times 3$ and $3 \times 5$ cases. Of course, this is only true here as we have both rotors at equal speed and the loading equally split between the two rotors.

Figure 14 shows that for the operative CROR configuration, the noise decreases with increasing fore and aft blade count, agreeing with previous parametric studies carried out by both the authors [20] and the work of Parry \& Vianello [19]. The exception to this occurs with equal blade counts as the acoustic interactions (i.e. constructive addition of the pressure signals) increases their noise levels. It is also shown that for the locked-fore configuration, the noise decreases for increasing aft blade count (i.e. the operative rotor).

Due to the increased noise level when fore and aft blade counts are equal, the fore-locked configuration has reduced noise levels compared to the operative configuration in this case. However, this may lead to unsuitable noise levels during cruise operations.

Figure 14 shows that the noise of the fore-locked configuration is generally lower than the operative configurations when $N_{2}>N_{1}$ (with the exception of the $6 \times 7$ configuration). In each case, the noise of each rotor for the operative configuration is less than the locked-fore case. However, the addition of the two sources results in an overall greater noise emission. When the fore blade count increases, the reduction in noise level, combined with the higher noise level of the aft-locked configuration (due to the increased loading), is such that the total noise is less than the locked-aft configuration.

Whilst the $4 \times 6$ combination was found to give the greatest gain for the fore-locked configuration 
compared to the operative configuration (around $3 \mathrm{~dB}(\mathrm{~A})$ ), the $6 \times 7$ combination gave the overall lowest noise levels. Therefore, CROR noise levels can be reduced by increasing the blade count without locking a blade row. However, the noise gains achieved by locking a blade row may be increased by increasing the aft blade count relative to the fore.

\subsection{Inoperative Aft Rotor}

The aft rotor is locked and trimmed to minimise torque whilst the fore rotor is trimmed to overcome the drag of the inoperative rotor plus the required thrust of the aircraft. The noise will be dominated by the self noise of the fore rotor. Along with the self noise, there will be interaction noise due to potential interaction from the aft rotor. There will also exist interaction noise from the aft rotor as the fore wake passes over the locked blades. Similarly to the locked-fore case, a number of CROR blade count combinations are investigated at take-off, climb-out and approach operating conditions (Table 3).

Figure 15 presents the computed noise for the locked-aft and baseline CROR configurations for all combinations of blade counts.

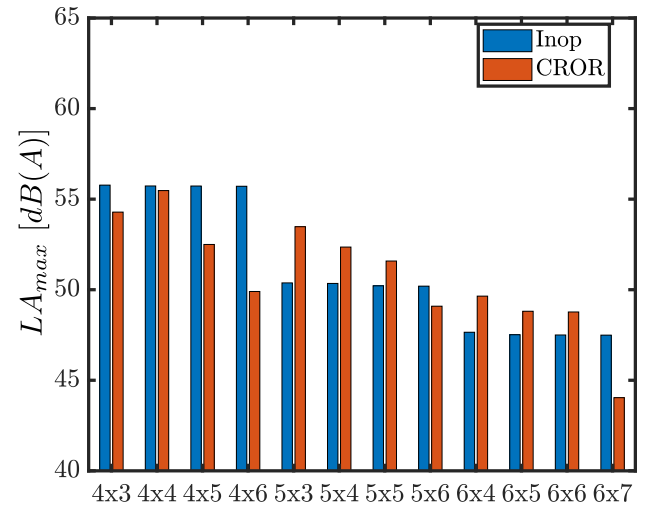

(a) Take-off

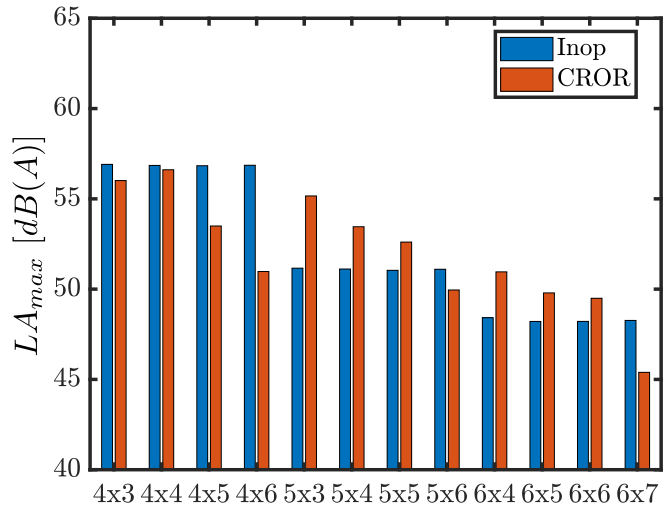

(b) Climb-out

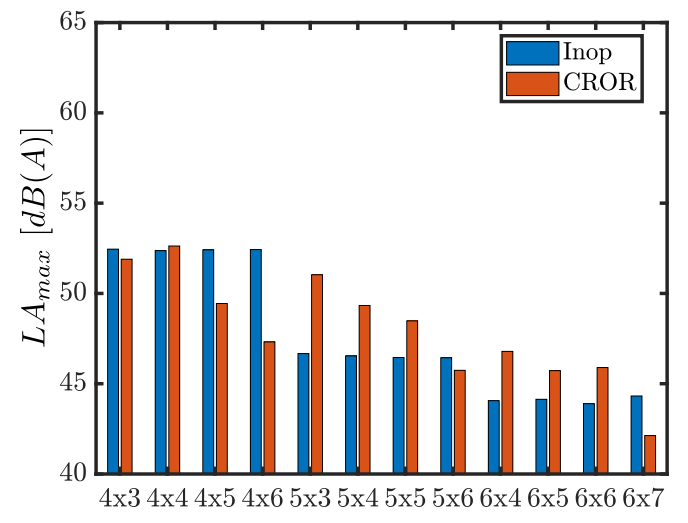

(c) Approach

Figure 15: Comparison of noise between baseline CROR and a CROR with locked-aft blade row. 
Comparing the two configurations, similarly to the locked-fore analysis, the trends appear consistent across all operating points. Again, as we are using the $L A_{\max }$ metric, the results are representative of steady loading noise which will dominate the flyover near the plane of rotation. Given the constant solidity, Figure 15 shows the locked-aft noise levels are consistent for the same fore blade count, i.e. the aft blade count has a negligible effect in this case.

No noise gains are observed for a locked-aft configurations with 4 fore blade $\left(N_{1}=4\right)$. Recall, differences in $L A_{\max }$ between the two configurations will be due to the acoustic effect of blade count and the difference in loading between the two configurations (i.e. thrust produced by a single or two rotors). As a result when $N_{1}=4$ the reduced loading on the operative configuration results in lower noise than the locked-aft configuration.

When the fore blade count of the locked-aft configuration is increased to 5 or 6 , the noise reduction due to the increased blade count is sufficient to offer gains compared to the operative configuration when $N_{2} \leq N_{1}$. For each case, the noise of each rotor of the operative configuration is less than the total for the locked-aft configuration. However, it is the addition of the two noise sources from each rotor that result in the greater noise levels in these cases. When $N_{2}>N_{1}$ the noise reduction of the aft rotor is such that the total noise of the operative configuration drops below the locked-aft configuration. Therefore, again we can say that the noise of the CROR can be significantly reduced by increasing either fore or aft blade count. In fact, the lowest noise level was produced by the $6 \times 7$ case of the operative configuration.

The greatest noise gains for the locked-aft configuration occur for the $5 \times 3$ combination at around $3 \mathrm{~dB}(\mathrm{~A})$ at take-off. Increasing the aft blade count further reduces these gains (as the operative CROR noise reduces). Therefore we can also say the noise gains of the locked-aft configuration can be increased by increasing the fore blade count relative to the aft (opposite to the locked-fore configuration).

Comparing Figures 14 and 15 , shows that locking either fore or aft rotor results in similar noise levels. Note comparisons are made on opposing blade pairs. For example the $4 \times 6$ case of the locked-fore configuration should be compared against he $6 \times 4$ case of the locked-aft configuration, etc. However, under closer inspection it is found that the locked-aft configuration has slightly higher noise levels. Recall, in using the $L A_{\max }$ metric, differences will be down to steady interactions that effect the self noise which will dominate near the plane of rotation. It was found that locking the fore rotor was beneficial to the operative aft rotor (due to the computed induced velocity lowering the inflow angle), requiring a lower setting angle to deliver the required thrust. Therefore, the locked-aft configuration resulted in a poorer aerodynamic performance and hence higher peak noise levels.

The maximum A-weighted SPL $\left(L A_{\max }\right)$ metric is used for general aviation aircraft certification [26], 
and this metric has been used to present the current results. However, CROR noise is highly directive and therefore, the $L A_{\max }$ metric may not be the most suitable when evaluating CROR noise. The Effective Perceived Noise Level (EPNL) is an integral metric that accounts for intensity and the duration of a noise source. Therefore, the EPNL metric is perhaps more suited for CROR analysis. To this end, Figure 16 compares the EPNL computed for the inoperative and operative CROR configurations at the take-off condition. The EPNL was calculated for a constant 1,000ft flyover.

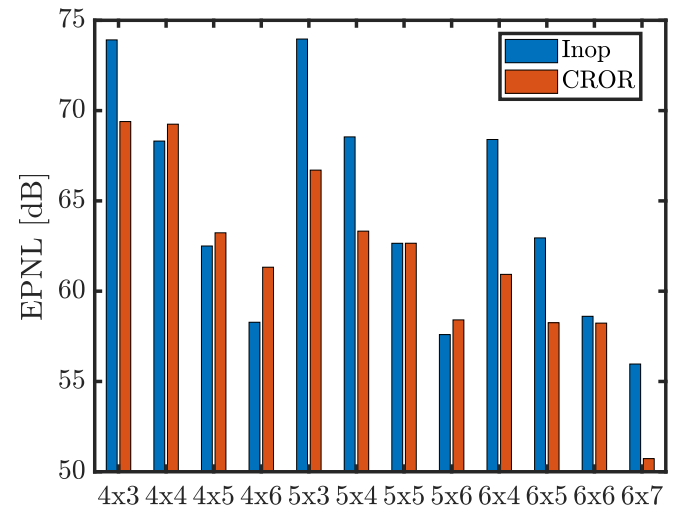

(a) Locked-fore

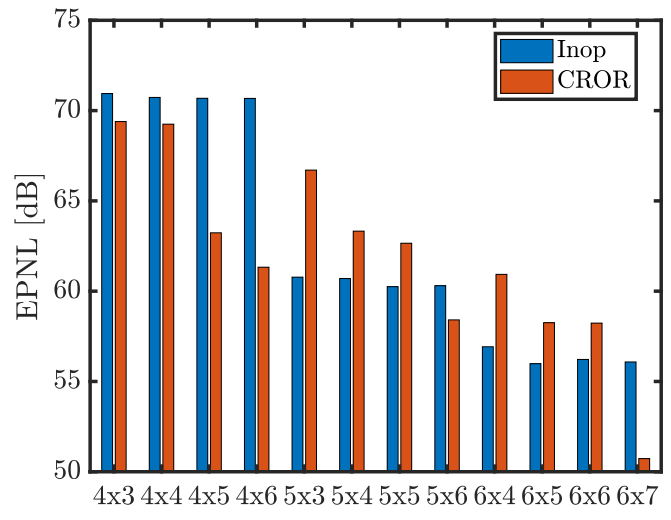

(b) Locked-aft

Figure 16: EPNL of baseline operative CROR and a CROR with a blocked blade row.

The use of the EPNL metric should allow for the significant CROR interactions leading to high noise near the plane of rotation to be accounted for. This is confirmed in Figure 16(a) where the trends differ from those when the $L A_{\max }$ metric was used. As discussed previously, the steady tones will radiate with the same efficiency across all configurations. Furthermore, there will be a number of interaction harmonics that will radiate very efficiently along the propeller axis (where $v=0$ ) for all configurations. The use of the EPNL metric should allow for us to account for these interaction tones that will increase noise levels across the directivity.

Using the EPNL metric, the differences in noise between configurations will be much more difficult to isolate. Again, the steady components will be important. Now however, the interaction components will also be significant. Figure 16(a) shows that there are now fewer locked-fore configurations producing less noise than the operative configurations. Namely, these are the $5 \times 5$ and $6 \times 6$ cases. Here, the locked-fore produces significant noise levels. These will results from the wake interactions from the locked fore rotor passing over the operative aft rotor.

Maximum gains for the locked-fore configuration again occur for the $4 \times 5$ case. Gains of around $3 \mathrm{~dB}$ (EPNL) are observed for this case. However, the $6 \times 7$ case for the operative configuration shows the lowest noise levels. Again it can therefore be said that CROR noise can be reduced significantly by 
increasing the blade count of fore and aft rotors.

Considering the locked-aft configuration, we now have the effect of upstream potential interactions being accounted for. However, Figure 16(b) shows consistent values across fore blade counts, thus indicating this component may be less significant when the aft rotor is locked. Therefore, using the EPNL metric, we see the same trends as using the $L A_{\max }$ metric. However, due to the interactions accounted for with the operative configuration, differences between the two configurations are now greater.

The $5 \times 3$ configuration again presents the greatest gain for the locked-aft configuration. Here, the gain is around $6 \mathrm{~dB}$ (EPNL) compared to the operative configuration. Again, the $6 \times 7$ configuration presents the lowest noise level for all configurations. From Figure 16(b), it may be again concluded that the locked-aft gains may be increased by increasing the fore blade count relative to the aft.

Due to the EPNL metric capturing a greater content of the noise signal, it highlights the differences in noise levels between the two cases compared to using the $L A_{\max }$ metric. Nonetheless, the $L A_{\max }$ metric is currently used for certification of light propeller driven aircraft and therefore, the current concepts must conform to the standards using this metric.

Locking of a blade row was found to decrease propulsive efficiency by around 5\%. Nonetheless, given the short duration of the take-off, climb-out and approach segments, the efficiency penalty may be worth the noise gains.

Whilst locking the aft rotor has been shown to reduce CROR noise, the noise gains are limited by the still present interactions with the locked row and the increased loading required to meet the aircraft thrust. In order to address these limitations, we investigate a number of additional configurations to further reduce CROR noise. Firstly we consider further removing the interactions by folding the aft rotor (Figure 1(d)]. Secondly, we reduce the loading on the fore rotor by increasing the diameter (Figure 1(e)]. The previous investigation showed little change in trend across operating points. Therefore, we now only consider the take-off operating condition (Table 3).

\subsubsection{Folded Aft Rotor}

In attempt to further reduce noise, the case of folding the aft rotor is next considered. In this configuration (Figure 1(d)], the aft rotor is locked and folded backwards for take-off and climb-out. During cruise, the rotor is then unfolded and operated to realise the efficiency gains of the CROR. During approach, the aft rotor is locked and again folded backwards. The folded rotor will eliminate both steady and unsteady interactions with the operative fore rotor. Furthermore, it should reduce the drag, and hence, the thrust 
requirements when compared to the locked case.

The folded rotor is modelled as an isolated SRP. Similarly to the previous cases, the rotor is trimmed to meet the required thrust and a fly-over is simulated for the calculation of $L A_{\max }$. Figure 17 shows a comparison of the noise computed for the locked-aft case and the folded aft case for all blade count combinations at take-off.

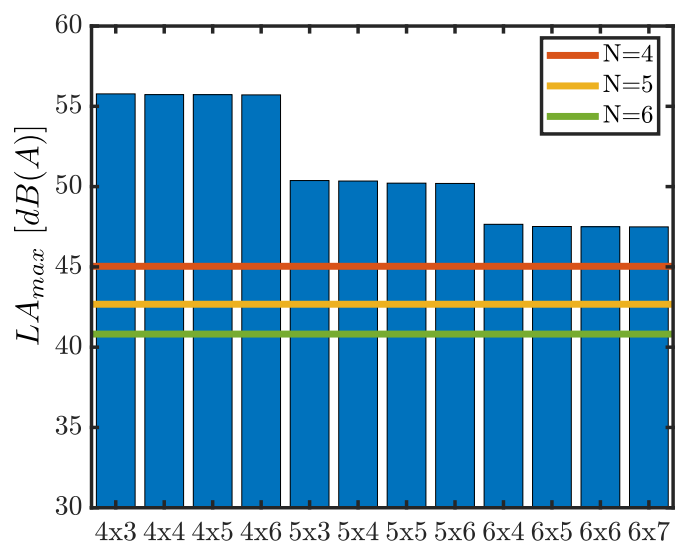

Figure 17: Comparison of total noise between locked-aft and folded-aft rotors for take-off conditions.

Figure 17 demonstrates that there is a significant noise gain when the locked-aft rotor is folded backwards. As the $L A_{\max }$ metric is used and as we compare the same operative rotor blade count, differences in noise between the two configuration will arise due to differences in loading. The folded aft rotor is modelled as an isolated SRP and hence there will be no interaction effects. On the other hand, for the unfolded case, there will steady interaction effects. In particular, the presence of the aft rotor will produce an induced velocity such that the operative rotor will require an increased setting angle to achieve the same thrust (and hence produce an increased drag component.) Furthermore, the locked rotor will produce a drag that will also have to be overcome by the operative rotor.

This additional loading results in an around $10 \mathrm{~dB}(\mathrm{~A})$ gain for the folded rotor. However, the gain decreases as the blade count is increased as the additional loading effect (from the induced component) decreases as the blade count is increased.

It is shown that folding the aft rotor can offer further noise gains when compared to the unfolded case. Again due to the minimal effect of the aft rotor, it can be designed independently of take-off requirements. When considering the system design, the additional weight and complexity of the folding mechanism must be considered against any noise gains achieved. 


\subsubsection{Increased Fore Radius}

We now consider further reducing the noise of the locked-aft configuration by increasing the fore rotor radius (whilst maintaining the tip speed). Note that all other spanwise distributions are the same as the baseline, only the tip radius is increased. Any gains will primarily be due to reduced blade element loading and the reduced rotational rate. Furthermore, the increased rotor radius will have a similar effect as clipping the aft rotor radius by reducing the tip vortex interaction with the aft rotor. However, we do not account for this noise component and hence will only investigate the effects of reduced loading.

For this study we increase the fore rotor radius by up to $30 \%$ whilst maintaining the tip speed by reducing the rotor rotation rate. Figure 18 shows the effect of increasing radius for all blade count combinations for both locked-aft and baseline CROR configurations.

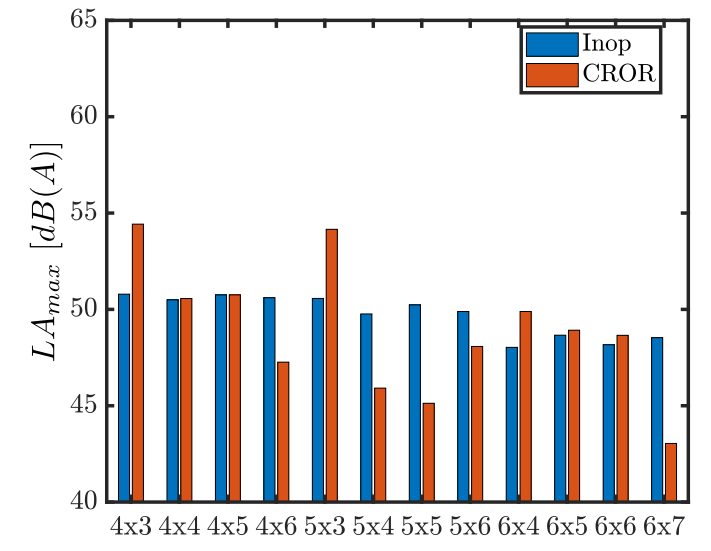

(a) $\Delta R_{1}=10 \%$

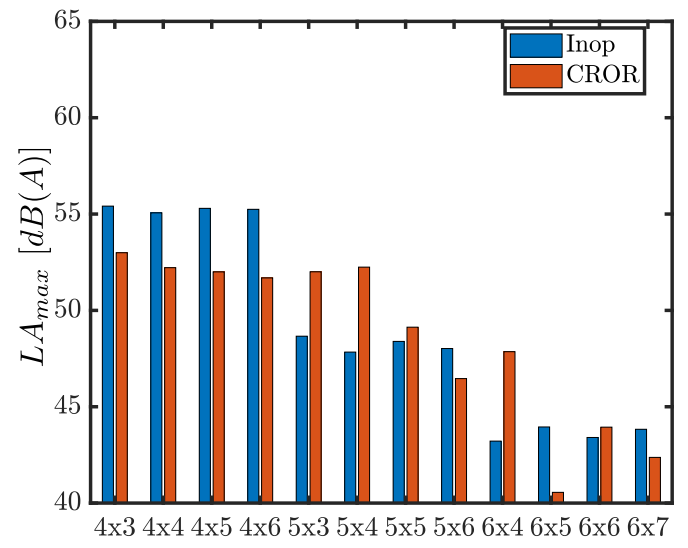

(b) $\Delta R_{1}=20 \%$

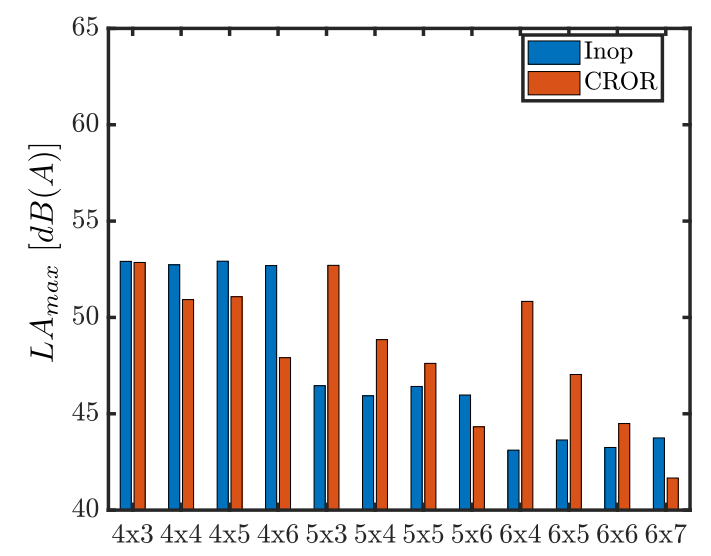

(c) $\Delta R_{1}=30 \%$

Figure 18: Effect of increasing radius on locked-aft and baseline CROR configurations.

The results demonstrate that for increasing tip radius, the noise generally reduces for both locked-aft and operative CROR configurations. For the operative CROR case, the noise for most configurations is 
dominated by the aft rotor. For increasing fore radius, there is a reduction in torque ratio between the two rotors. This is known to have an important role in the emitted noise [18], and therefore this will also limit noise reductions. Furthermore, the loading is already half of that compared to the locked-aft case and changes to loading will be less significant. Combined with the fact that the aft rotor is the dominant noise source, the effects of increased fore radius will be less pronounced. This all results in a more complex trend for the operative CROR configuration with noise increases for some combinations between the increased tip and baseline configurations.

When the fore blade count is equal to 4 , the noise of the locked-aft configuration substantially decreases when the tip radius is extended by $10 \%$. The noise level then increases for a $20 \%$ tip increase before reducing slightly for a $30 \%$ increase in tip radius. When the fore blade count is equal to 5 or 6 , increasing the tip radius results in a continual decrease in noise levels.

When the tip radius increases, the number of blade count combinations showing a noise gain increases. The most substantial gains are found when the tip radius is extended by $30 \%$. In fact, when the tip is extended by this much, there are noise gains for all but one combination with fore blade counts of 5 and 6. Figure 19 shows the difference in noise levels between operative and aft-locked configurations for the $6 \times 4$ configurations for a range of increasing tip radius (note, negative indicates noise gains over operative configuration). The markers indicate the computed values with the solid line used to show the trend. Figure 19 shows that up to almost $8 \mathrm{~dB}(\mathrm{~A})$ can be gained with a locked-fore configuration with a

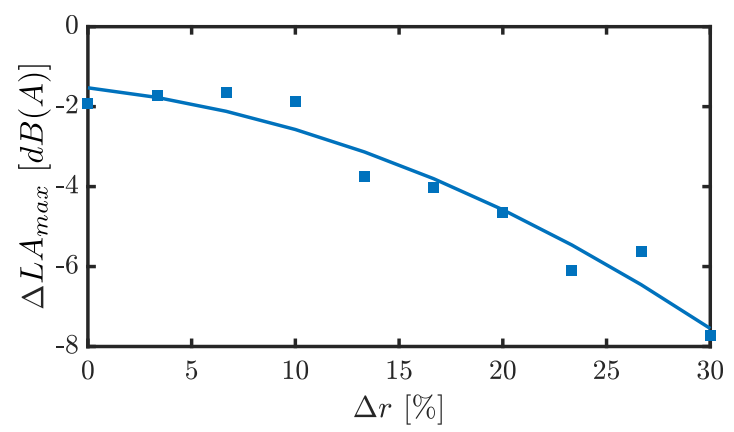

Figure 19: Difference in noise between locked-aft and operative CROR for increasing radius for the $6 \times 4$ combination.

$30 \%$ tip increase, compared to an operative configuration with the same tip extension.

When the tip radius is increased, the propulsive efficiency was found to reduce significantly. For a 10 percent increase in radius, the efficiency was seen to drop by around the same level. With the increasing radius, the rotor is less loaded and moves further away from the optimal lift-drag ratio. With the CROR being designed with an increased diameter to accommodate single rotor operation, further increases in 
diameter result in a rapid reduction in efficiency.

Increasing the tip radius has shown the potential to significantly reduce CROR noise with the aft rotor locked. However, this reduced noise comes with a significant penalty to the propulsive efficiency. Additional gains are envisioned by reducing tip vortex interaction.

\section{Conclusions}

This contribution has presented a number of novel CROR configurations aimed at reducing CROR noise at take-off, climb-out, and approach conditions for general aviation class aircraft. Each concept was investigated over a range of fore and aft blade count combinations in order to cover a wide design space. The results obtained have demonstrated that the novel concepts proposed can be used to reduce CROR noise.

First, the locking of a blade row was considered. It was demonstrated that locking either fore or aft blade row resulted in noise gains for a number of blade count combinations. Throughout the analysis the solidity was held constant to ensure predictions were purely from an acoustic point of view.

When locking a blade row, noise gains are limited due to the increased loading on the rotor required to deliver the total thrust. When the fore blade row was locked, a maximum noise reduction of $3 \mathrm{~dB}(\mathrm{~A})$ or $3 \mathrm{~dB}$ (EPNL) was observed for the $4 \times 6$ blade count configuration. When the aft rotor was locked, a maximum noise gain of around $3 \mathrm{~dB}(\mathrm{~A})$ or $6 \mathrm{~dB}$ (EPNL) were observed. However, noise was not reduced for every combination and for a number of blade counts, the locking of a rotor resulted in increased noise levels. In fact, the $6 \times 7$ case for the operative CROR produced the lowest overall noise levels. The results demonstrated that CROR noise could be significantly reduced by increasing the blade count. The noise gains for the locked-fore configuration could be increased by increasing the aft blade count relative to the fore. For the locked-aft configuration, noise gains could be increased by increasing the fore blade count relative to the aft.

When locking a blade row, an efficiency penalty of around 5\% resulted for both configurations. Therefore, this penalty must be weighed against any noise gains. Furthermore, the analysis was carried out from a purely acoustical point of view, and a more multi-disciplinary approach will be required to bring the concept further.

In order to investigate further noise gains, the locked-aft rotor was then folded backwards. It was demonstrated that significant gains could be made over both the aft-locked and operative configurations. Locking the aft rotor and folding it backwards resulted in a maximum gain of around $12 \mathrm{~dB}(\mathrm{~A})$ when 
compared to the operative configuration using a $5 \times 3$ blade count. Again a further efficiency penalty was associated to this acoustic gain.

The locked-aft configuration gains are limited due to the increased loading. It was found that increasing the tip radius, and hence unloading the rotor (and reducing the tip speed) resulted in reduction in noise when compared to a operative CROR with the same tip extension. Results indicated that 8 $\mathrm{dB}(\mathrm{A})$ could be saved by increasing the tip radius by $30 \%$. However, this noise came at a significant efficiency penalty, even for smaller increases in rotor diameter.

To conclude, the novel CROR configurations presented have shown the ability to reduce CROR noise in the terminal area. The noise can be reduced by as much as $12 \mathrm{~dB}(\mathrm{~A})$ by locking the aft rotor and folding it backwards for take-off. These noise gains come at the cost of a small efficiency penalty. However, due to the short duration the aircraft would operate with this configuration and the efficiency gains achievable by utilising the aft rotor in cruise, this is deemed acceptable. It is important to note that the noise reduction is purely from acoustic criteria. Therefore, each case must be carefully considered for its aerodynamic and structural performance too. Furthermore, these combinations must be carefully considered for their noise and aerodynamic performance at cruise. To proceed, future analysis should consider a multi-disciplinary approach, and with the use of low-order models, a suitable optimal design can be developed. Prior to this, the predictions of these novel concepts should be validated to confirm the findings presented throughout this work, particularly as a number of combinations increased the noise relative to the operative CROR. Further, in our analysis we performed only axial flight simulations of the CROR noise sources. Future work is recommended to account for the various other aircraft sources to develop a clearer indication of the performance of the above concepts. A more realistic flight path should also be simulated to give a more substantial representation of the noise sources in the terminal areas.

\section{Acknowledgements}

This research is supported by a private sponsorship (Mr Mike Newton) and a UK Research Council Grant EP/S010092/1 (Methods and Experiments for Novel Rotorcraft).

\section{References}

[1] A. Abbas, J. De Vicente, and E. Valero, "Aerodynamic technologies to improve aircraft performance," Aerospace Science and Technology, vol. 28, no. 1, pp. 100-132, 2013. [Online]. Available: http://dx.doi.org/10.1016/j.ast.2012.10.008 
[2] D. E. V. Zante, "Progress in Open Rotor Research: A U.S. Perspective," in ASME Turbo Expo 2015, Montréal, Canada, 2015, pp. 1-14.

[3] F. Lanchester, "Contra-Props," Flight Magazine, pp. 418-419, 1941.

[4] W. A. Bartlett Jr., "Wind-tunnel Tests of a Dual-rotating Propeller Having One Component Locked or Windmilling," NACA ARR L5A13a, 1945.

[5] F. W. Caldwell, "Counter-Rotating Propeller," U.S. Patent 2,312,624, 1943.

[6] E. Burton, "Multiengine Contra-Rotating Propeller Drive Transmission," U.S. Patent 2,581,320, 1952.

[7] A. Zachariadis, C. Hall, and A. B. Parry, "Contrarotating Open Rotor Operation for Improved Aerodynamics and Noise at Takeoff," Journal of Turbomachinery, vol. 135, no. 031010, 2013. [Online]. Available: https://doi.org/10.1115/1.4006778

[8] C. Weckmüller and S. Guérin, "On the influence of trailing-edge serrations on open-rotor tonal noise," in 18th AIAA/CEAS Aeroacoustics Conference, 2012. [Online]. Available: https://doi.org/10.2514/6.2012-2124

[9] A. Stürmer and R. A. Akkermans, "Multidisciplinary analysis of CROR propulsion systems: DLR activities in the JTI SFWA project," CEAS Aeronautical Journal, vol. 5, no. 3, pp. 265-277, 2014.

[10] A. Stuermer, J. Yin, and R. Akkermans, "Progress in aerodynamic and aeroacoustic integration of CROR propulsion systems," Aeronautical Journal, vol. 118, no. 1208, pp. 1137-1158, 2014.

[11] R. A. D. Akkermans, A. Stuermer, and J. W. Delfs, "Active Flow Control for Interaction Noise Reduction of Contra-Rotating Open Rotors," AIAA Journal, vol. 54, no. 4, pp. 1413-1423, 2016. [Online]. Available: http://dx.doi.org/10.2514/1.J053756

[12] B. Shivashankara, J. D., and R. Cuthberston, "Installation effects on counter rotating propeller noise," in 13th Aeroacoustics Conference, Tallahassee, FL., 1990. [Online]. Available: https://doi.org/10.2514/6.1990-4023

[13] J. Ricouard, E. Julliard, M. Omais, V. Regnier, A. B. Parry et al., "Installation Effects on Contra-Rotating Open Rotor Noise," 16th AIAA/CEAS Aeroacoustics Conference, no. August 2015, pp. 1-8, 2010. [Online]. Available: http://dx.doi.org/10.2514/6.2010-3795

[14] N. G. Sohoni, C. A. Hall, and A. B. Parry, "The influence of an upstream pylon on open rotor aerodynamics at angle of attack," Journal of Turbomachinery, no. c, pp. 1-14, 2018. [Online]. Available: http://turbomachinery.asmedigitalcollection.asme.org/article.aspx?doi= 10.1115/1.4041082

[15] A. Peters and Z. S. Spakovszky, "Rotor Interaction Noise in Counter-Rotating Propfan Propulsion Systems," Journal of Turbomachinery, vol. 134, no. 1, pp. 2821-2834, 2010. [Online]. Available: http://proceedings.asmedigitalcollection.asme.org/proceeding.aspx?articleid=1610274

[16] C. K. W. Tam, M. Salikuddin, and D. B. Hanson, "Acoustic Interference of Counter-Rotating Propellers," Journal of Sound and Vibration, vol. 124, no. 2, pp. 357-366, 1988.

[17] R. A. D. Akkermans, J. W. Delfs, C. O. Márquez, A. Stuermer, C. Richter et al., "Aeroacoustic and Aerodynamic Importance of Unequal Rotor Rotation Speeds of a CROR," New Results in Numerical and Experimental Fluid Mechanics IX, vol. 124, 2014.

[18] G. Delattre and F. Falissard, "Influence of Torque Ratio on Counter-Rotating Open-Rotor Interaction Noise," AIAA Journal, vol. 53, no. 9, pp. 2726-2738, 2015. [Online]. Available: http://arc.aiaa.org/doi/10.2514/1.J053797 
[19] A. B. Parry and S. Vianello, "A project study of open rotor noise," International Journal of Aeroacoustics, vol. 11, no. 2, pp. 247-258, 2012.

[20] D. A. Smith, A. Filippone, and N. Bojdo, "A Parametric Study of Counter Rotating Open Rotor Noise," in 25th AIAA/CEAS Aeroacoustics Conference Downloaded, no. May, Delft, The Netherlands, 2019.

[21] G. N. Barakos and C. S. Johnson, "Acoustic comparison of propellers," International Journal of Aeroacoustics, vol. 15, no. 6-7, pp. 575-594, 2016.

[22] G. Chirico, G. N. Barakos, and N. Bown, "Numerical aeroacoustic analysis of propeller designs," Aeronautical Journal, vol. 122, no. 1248, pp. 283-315, 2018.

[23] D. A. Smith, A. Filippone, and N. Bojdo, "Low Order Multidisciplinary Optimisation of CounterRotating Open Rotors," in European Rotorcraft Forum, Delft, 2018.

[24] D. B. Hanson, “Noise of Counter-rotation Propellers," Journal of Aircraft, vol. 22, no. 7, pp. 609$617,1985$.

[25] A. B. Parry, "Theoretical Prediction of Counter-Rotating Propeller Noise," PhD Thesis, University of Leeds, 1988.

[26] International Civil Aviation Organization, "CHAPTER 10. Propeller-driven aeroplanes not exceeding 8618 kg," in ICAO Annex 16: Environmental Protection Volume 1 - Aircraft Noise, 2011.

[27] W. Z. Stepniewski, "Rotary-wing Aerodynamics - Volume I: Basic Theories of Rotor Aerodynamics (with Application to Helicopters)," Rotary-Wing Aerodynamics, vol. I, 1979.

[28] R. D. Harrington, "Full-Scale-Tunnel Investigation of the Static-Thrust Performance of a Coaxial Helicopter Rotor," NACA TN 2318, 1951.

[29] A. Filippone, "Rapid Estimation of Airfoil Aerodynamics for Helicopter Rotors," Journal of Aircraft, vol. 45, no. 4, pp. 1468-1472, 2008. [Online]. Available: https://doi.org/10.2514/1.35560

[30] J. J. Moré, “The Levenberg-Marquardt Algorithm: Implementation and Theory," in Lecture Notes in Mathematics 630. Springer Verlag, 1977, pp. 105-116.

[31] M. Abramowitz and I. A. Stegun, Handbook of Mathematical Functions, 10th ed. Washington D.C: National Bureau of Standards, 1964.

[32] R. J. Jeracki, D. C. Mikkelson, and B. J. Blaha, "Wind Tunnel Performance of Four Energy Efficient Propellers Designed for Mach 0.8 Cruise," NASA TM-79124, 1979.

[33] R. D. Hager and D. Vrabel, “Advanced Turboprop Project,” NASA SP-495, 1988.

[34] D. Dunham, C. L. Gentry, and P. L. Coe, "Low-Speed Wind-Tunnel Tests of Single- and CounterRotation Propellers," NASA TM-87656, 1986.

[35] D. B. Hanson, "Helicoidal Surface Theory for Harmonic Noise of Propellers in the Far Field," AIAA Journal, vol. 18, no. 10, pp. 1213-1220, 1980.

[36] F. Farassat, "Linear Acoustic Formulas for Calculation of Rotating Blade Noise," AIAA Journal, vol. 19, no. 9, pp. 1122-1130, 1981. [Online]. Available: https://doi.org/10.2514/3.60051

[37] B. Magliozzi, D. B. Hanson, and R. K. Amiet, "Propeller and Propfan Noise," NASA Langley Research Center, Aeroacoustics of Flight Vehicles: Theory and Practice. Volume 1: Noise Sources, 1991. 
[38] D. B. Hanson, "Influence of Propeller Design Parameters on Far-Field Harmonic Noise in Forward Flight," AIAA Journal, vol. 18, no. 11, pp. 1313-1319, 1980.

[39] A. B. Parry and D. G. Crighton, "Prediction of Counter-Rotation Propeller Noise," in AIAA 12th Aeroacoustics Conference, San Antonio, TX, 1989.

[40] A. B. Parry, "Modular Prediction Scheme for Blade Row Interaction Noise," Journal of Propulsion and Power, vol. 13, no. 3, pp. 334-341, 1997.

[41] M. J. Kingan and R. H. Self, "Counter-Rotation Propeller Tip Vortex Interaction Noise," 15th AIAA/CEAS Aeroacoustics Conference (30th AIAA Aeroacoustics Conference), no. May, pp. 11-13, 2009. [Online]. Available: http://arc.aiaa.org/doi/abs/10.2514/6.2009-3135

[42] V. P. Blandeau and P. F. Joseph, "A Semi-Analytical Model for the Prediction of Broadband Noise Due to Rotor-Wake Interaction in Contra-Rotating Propfans," 16th International Congress on Sound and Vibration, no. July 2009, pp. 5-9, 2009.

[43] V. P. Blandeau and P. F. Joseph, "Broadband Noise Due to Rotor-Wake/Rotor Interaction in Contra-Rotating Open Rotors," AIAA Journal, vol. 48, no. 11, pp. 2674-2686, 2010. [Online]. Available: http://arc.aiaa.org/doi/10.2514/1.J050566

[44] M. J. Kingan, "Open Rotor Broadband Interaction Noise," Journal of Sound and Vibration, vol. 332, no. 17, pp. 3956-3970, 2013.

[45] P. J. W. Block, "Installation Noise Measurements of Model SR and CR Propellers," NASA TM $85790,1984$.

[46] P. J. W. Block, "Noise radiation patterns of counter-rotation and unsteadily loaded single-rotation propellers," Journal of Aircraft, vol. 22, no. 9, pp. 776-783, 1985. [Online]. Available: http://arc.aiaa.org/doi/abs/10.2514/3.45201

[47] P. J. W. Block, R. J. Klatte, and P. M. Druez, "Counter-Rotating Propeller Noise Directivity and Trends," in AIAA 10th Aeroacoustics Conference, Seattle, Washington, 1986. [Online]. Available: https://doi.org/10.2514/6.1986-1927

[48] A. Filippone and Z. Mohamed-Kassim, "Multi-disciplinary simulation of propeller-turboprop aircraft flight," Aeronautical Journal, vol. 116, no. 1184, pp. 985-1014, 2012. [Online]. Available: https://doi.org/10.1017/S0001924000007454

[49] A. Filippone, "Aircraft Noise: Noise Sources," in Advanced Aircraft Flight Performance. Cambridge University Press, 2012, pp. 470-532. [Online]. Available: http://dx.doi.org/10.1017/ CBO9781139161893 